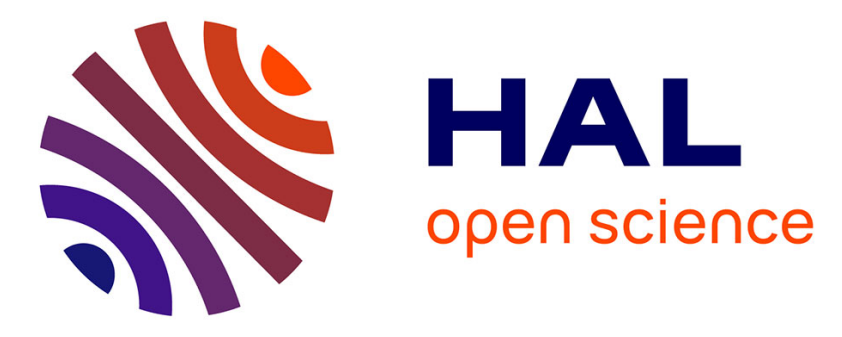

\title{
Genomic imprinting mediates dosage compensation in a young plant XY system.
}

\author{
Aline Muyle, Niklaus Zemp, Cécile Fruchard, Radim Cegan, Jan Vrána, \\ Clothilde Deschamps, Raquel Tavares, Roman Hobza, Franck Picard, Alex \\ Widmer, et al.
}

\section{To cite this version:}

Aline Muyle, Niklaus Zemp, Cécile Fruchard, Radim Cegan, Jan Vrána, et al.. Genomic imprinting mediates dosage compensation in a young plant XY system.: An article peer-reviewed and recommended by Peer Community In Evolutionary Biology (PCI Evol Biol). 2018. hal-01851376

\section{HAL Id: hal-01851376 https://hal.science/hal-01851376}

Preprint submitted on 30 Jul 2018

HAL is a multi-disciplinary open access archive for the deposit and dissemination of scientific research documents, whether they are published or not. The documents may come from teaching and research institutions in France or abroad, or from public or private research centers.
L'archive ouverte pluridisciplinaire HAL, est destinée au dépôt et à la diffusion de documents scientifiques de niveau recherche, publiés ou non, émanant des établissements d'enseignement et de recherche français ou étrangers, des laboratoires publics ou privés. 
bioRxiv preprint first posted online Aug. 21, 2017; doi: http://dx.doi.org/10.1101/179044. The copyright holder for this preprint (which was not peer-reviewed) is the author/funder. All rights reserved. No reuse allowed without permission.

\section{5: Peer Community In}

Muyle A, Zemp N, Fruchard C, Cegan R, Vrana J, Deschamps C, Tavares R, Picard F, Hobza R, Widmer A and Marais G. 2018. Genomic imprinting mediates dosage compensation in a young plant $\mathbf{X Y}$ system. bioRxiv. bioRxiv, 179044, https://doi.org/10.1101/179044

An article reviewed and recommended by Peer Community In Evolutionary Biology:

http://dx.doi.org/10.24072/pci.evolbiol.100044 


\section{Genomic imprinting mediates dosage compensation in a young plant XY}

3 Authors: Aline Muyle ${ }^{1^{*}}$, Niklaus Zemp ${ }^{2,6^{*}}$, Cécile Fruchard ${ }^{1}$, Radim Cegan ${ }^{3}$, Jan Vrana ${ }^{4}$,

4 Clothilde Deschamps ${ }^{5}$, Raquel Tavares ${ }^{1}$, Roman Hobza ${ }^{3,4}$, Franck Picard ${ }^{1}$, Alex Widmer ${ }^{6}$, Gabriel

5 AB Marais $^{1}$

\section{Affiliations:}

$7 \quad{ }^{1}$ Laboratoire “Biométrie et Biologie Evolutive”, CNRS / Université Lyon 1, France.

$8 \quad{ }^{2}$ Genetic Diversity Center (GDC), ETH Zurich, Switzerland.

$9 \quad{ }^{3}$ Institute of Biophysics of the Czech Academy of Sciences, Brno.

$10{ }^{4}$ Institute of Experimental Botany, Center of the Hana Region for Biotechnological and

11 Agricultural Research, Olomouc, Czech Republic

$12{ }^{5}$ Center for Bioinformatics in Rhônes-Alpes (PRABI), Lyon, France

$13{ }^{6}$ Institute of Integrative Biology, ETH Zurich, Switzerland.

$14{ }^{*}$ These authors contributed equally to this work. 
This preprint has been reviewed and recommended by Peer Community In Evolutionary

Biology (http://dx.doi.org/10.24072/pci.evolbiol.100044).

Sex chromosomes have repeatedly evolved from a pair of autosomes ${ }^{1}$. Consequently, $\mathrm{X}$ and Y chromosomes initially have similar gene content, but ongoing $\mathrm{Y}$ degeneration leads to reduced Y gene expression and eventual Y gene loss. The resulting imbalance in gene expression between $\mathrm{Y}$ genes and the rest of the genome is expected to reduce male fitness, especially when protein networks have components from both autosomes and sex chromosomes. A diverse set of dosage compensating mechanisms that alleviates these negative effects has been described in animals ${ }^{2-4}$. However, the early steps in the evolution of dosage compensation remain unknown and dosage compensation is poorly understood in plants ${ }^{5}$. Here we show a novel dosage compensation mechanism in the evolutionarily young XY sex determination system of the plant Silene latifolia. Genomic imprinting results in higher expression from the maternal $\mathrm{X}$ chromosome in both males and females. This compensates for reduced Y expression in males but results in $\mathrm{X}$ overexpression in females and may be detrimental. It could represent a transient early stage in the evolution of dosage compensation. Our finding has striking resemblance to the first stage proposed by Ohno for the evolution of $\mathrm{X}$ inactivation in mammals. complete dosage compensation through both ancestral expression recovery in males and equal expression between the sexes (hereafter sex equality) ${ }^{6}$. In Caenorhabditis elegans, both $\mathrm{X}$ chromosomes are downregulated in XX hermaphrodites resulting in sex equality, but only a few genes have their $\mathrm{X}$ expression doubled for ancestral expression recovery ${ }^{7}$. In placental mammals, including humans, one $\mathrm{X}$ chromosome is randomly inactivated in $\mathrm{XX}$ females, resulting in sex equality but without recovering the ancestral expression of sex chromosomes, except for a few 
38 dosage-sensitive genes whose $\mathrm{X}$ expression was doubled in both sexes ${ }^{8-12}$. In the marsupials, the

39 paternal X chromosome is consistently inactivated in XX females ${ }^{13}$. Differential expression that

40 depends on the parent of origin is known as genomic imprinting ${ }^{14}$, and this mechanism also

41 operates in the mouse placenta ${ }^{15}$.

42 Despite the plethora of studies on gene expression on sex chromosomes, it is not yet clear

43 if genomic imprinting is commonly involved in the early steps of dosage compensation

44 evolution. In a seminal work, Ohno hypothesized a two-step process for the evolution of dosage

45 compensation ${ }^{16}$. In the first step, expression from the $\mathrm{X}$ is doubled, thereby mediating the

46 recovery of ancestral expression in $\mathrm{XY}$ males. Second, the resulting overexpression in $\mathrm{XX}$

47 females selects for $\mathrm{X}$ inactivation. This scenario is consistent with the fact that sexual selection is

48 often stronger on males than on females. Under this scenario, selection on $\mathrm{XY}$ males to

49 upregulate their single $\mathrm{X}$ chromosome should be stronger than selection on females, leading to

50 overexpression in females until a second correcting mechanism evolves ${ }^{3}$. However, in order to

51 understand these early steps of dosage compensation evolution, species with young sex

52 chromosomes must be studied.

53 The plant Silene latifolia is an ideal model to study early steps of sex chromosome

54 evolution thanks to its pair of $\mathrm{X} / \mathrm{Y}$ chromosomes that evolved $\sim 4 \mathrm{Mya}^{17}$. Dosage compensation is

55 poorly understood in plants ${ }^{5}$. Thus far only sex equality has been studied. Equal expression

56 levels were observed for males and females for some genes despite $\mathrm{Y}$ expression degeneration ${ }^{18-}$

$57{ }^{23}$. However, the mechanisms through which sex equality is achieved - and whether ancestral

58 expression is recovered in S. latifolia males - remain unknown. To address these questions, we

59 have developed an approach relying on (i) the use of an outgroup without sex chromosomes as

60 an ancestral autosomal reference ${ }^{5}$ in order to determine whether $\mathrm{X}$ chromosome expression 
61 increased or decreased in S. latifolia, (ii) the application of methods to study allele-specific

62 expression while correcting for reference mapping bias ${ }^{5}$, and (iii) a statistical framework to 63 quantify dosage compensation ${ }^{5}$.

64 Because only $\sim 25 \%$ of the large and highly repetitive S. latifolia genome has been 65 assembled so $\mathrm{far}^{23}$, we used an RNA-seq approach based on the sequencing of a cross (parents 66 and a few offspring of each sex), to infer sex-linked contigs (i.e. contigs located on the non67 recombining region of the sex chromosome pair $)^{24}$. X/Y contigs show both $\mathrm{X}$ and $\mathrm{Y}$ expression, 68 while $\mathrm{X}$-hemizygous contigs are $\mathrm{X}$-linked contigs without $\mathrm{Y}$ allele expression. We made 69 inferences separately for three tissues: flower buds, seedlings and leaves (Supplementary Table 70 S2). Results are consistent across tissues and flower buds and leaves are shown in 71 Supplementary Materials. In seedlings, 1100 sex-linked contigs were inferred. Among these, $7215 \%$ of contigs with significant expression differences between males and females were removed 73 for further analyses (Supplementary Table S2 and Materials and Methods). These are likely 74 involved in sex-specific functions and are not expected to be dosage compensated ${ }^{25}$. This was 75 done as a usual procedure for studying dosage compensation, however the resulting trends and 76 significance levels are not affected. About half of the non sex-biased sex-linked contigs could be 77 validated by independent data using three sources: literature, a genetic map and sequence data 78 from Y flow-sorted chromosomes (see Supplementary Table S2 and Materials and Methods). X79 hemizygous contigs are more difficult to identify than $\mathrm{X} / \mathrm{Y}$ contigs using an RNA-seq approach 80 (see Supplementary Text S1). This explains conflicting earlier results on dosage compensation in 81 S. latifolia 5 . A study using genomic data (i.e. not affected by the aforementioned ascertainment 82 bias) found sex-equality in approximately half of the studied X-hemizygous genes ${ }^{23}$. In our set of 83 X-hemizygous contigs, no evidence for dosage compensation was found (Supplementary Text 
84 S1), in agreement with previous work relying on an RNA-seq approach ${ }^{18,22}$. This could be due to 85 an over-representation of dosage insensitive genes in our set of $\mathrm{X}$-hemizygous contigs 86 (Supplementary Text S1). We estimated paternal and maternal allele expression levels in males and females for sex88 linked and autosomal contigs in S. latifolia after correcting for reference mapping bias (Materials 89 and Methods). We then compared these allelic expression levels to one or two closely related 90 outgroups without sex chromosomes in order to polarise expression changes in S. latifolia. For 91 autosomal contigs, expression levels did not differ between S. latifolia and the outgroups (Figure 92 1). This is due to the close relatedness of the outgroups ( 5My, Supplementary Figure S1), and 93 validates their use as a reference for ancestral expression levels. We used the ratio of Y over $\mathrm{X}$ 94 expression levels in S. latifolia males as a proxy for $\mathrm{Y}$ degeneration and then grouped contigs on 95 this basis. As expression of the $\mathrm{Y}$ allele decreased (paternal allele in blue in Figure 1), expression 96 of the corresponding $X$ allele in males increased (maternal allele in red in Figure 1). This is the 97 first direct evidence for ancestral expression recovery in S. latifolia, i.e. ancestral expression 98 levels are reestablished in males despite $\mathrm{Y}$ expression degeneration. In females, expression of the 99 maternal $\mathrm{X}$ allele also increased with $\mathrm{Y}$ degeneration (gray bars in Figure 1), similarly to the 100 maternal $\mathrm{X}$ allele in males. The paternal $\mathrm{X}$ alleles in females, however, maintained ancestral 101 expression levels, regardless of $\mathrm{Y}$ degeneration (black bars in Figure 1). Consequently, sex 102 equality is not achieved in S. latifolia due to upregulation of sex-linked genes in females 103 compared to ancestral expression levels. These results were confirmed in two other tissues and 104 when analysing independently validated contigs only (although statistical power is sometimes 105 lacking due to the limited number of validated contigs, Supplementary Figures S2-S7). 
107 and Supplementary Figures S2-S7) establishes a role for genomic imprinting in dosage 108 compensation. In order to statistically test this inference at the SNP level, we used a linear 109 regression model with mixed effects (Materials and Methods). Outgroup species were used as a 110 reference and expression levels in S. latifolia were then analyzed while accounting for the 111 variability due to contigs and individuals. The joint effect of the parental origin and the 112 degeneration level was estimated, which allowed computing expression differences between 113 maternal and paternal alleles in females for different $\mathrm{Y} / \mathrm{X}$ degeneration categories (Figure 2).

114 Maternal and paternal alleles of autosomal SNPs were similarly expressed in females, indicating 115 a global absence of genomic imprinting for these SNPs. However, for X/Y SNPs, the difference 116 between the maternal and paternal $\mathrm{X}$ in females increased with $\mathrm{Y}$ degeneration. These results 117 were confirmed in two other tissues and when analysing independently validated contigs only 118 (although statistical power is sometimes lacking due to the limited number of validated contigs, 119 Supplementary Figures S8-S13).

120 Previous studies that showed sex equality in S. latifolia could have been explained by 121 simple buffering mechanisms, where one copy of a gene is expressed at a higher level when 122 haploid than when diploid, due to higher availability of the cell machinery or adjustments in gene 123 expression networks ${ }^{23,26,27}$. However, the upregulation of the $\mathrm{X}$ chromosome we reveal here in $S$. 124 latifolia males cannot be explained by buffering mechanisms alone, as the maternal $\mathrm{X}$ in females 125 would otherwise not be upregulated. Instead, our findings indicate that a specific dosage 126 compensation mechanism relying on genomic imprinting has evolved in S. latifolia. This 127 apparent convergent evolution with marsupials is mediated by different mechanisms (in 128 marsupials the paternal X is inactivated ${ }^{13}$, while in S. latifolia the maternal X is upregulated). 
An exciting challenge ahead will be to understand how upregulation of the maternal $\mathrm{X}$ is

130 achieved in S. latifolia males and females at the molecular level. Chromosome staining suggests

131 that DNA methylation is involved. Indeed, one arm of one of the two X chromosomes in females

132 was shypomethylated, as well as the same arm of the single $\mathrm{X}$ in males ${ }^{28}$ (Figure 3 and

133 Supplementary Figure S14). Based on our results, we hypothesize that the hypomethylated X

134 chromosome corresponds to the maternal, upregulated X. Unfortunately, parental origin of the X

135 chromosomes was not established in this study ${ }^{28}$. It would be of interest in the future to study

136 DNA methylation patterns in S. latifolia paternal and maternal X chromosomes, along with the

137 homologous pair of autosomes in a closely related species without sex chromosomes. The

138 methylation pattern observed by chromosome staining suggests that dosage compensation in $S$.

139 latifolia could be a chromosome arm-wide phenomenon. To test this hypothesis with expression

140 data, positions of genes along the $\mathrm{X}$ chromosome remain to be elucidated.

141 Our study is the first to establish female upregulation of the $\mathrm{X}$ chromosome compared to

142 autosomes, as predicted by Ohno. An earlier report in Tribolium castaneum was later shown to

143 be due to biases from inclusion of gonads in whole body extracts ${ }^{4}$. X overexpression in females

144 may be deleterious. Its occurence suggests that reduced expression of sex-linked genes in males

145 is more deleterious than overexpression in females. This potentially suboptimal situation may be

146 transitory and a consequence of the young age of S. latifolia sex chromosomes. Sex equality may

147 evolve at a later stage, following the evolutionary path trajectory originally proposed by Ohno

148 for placental mammals ${ }^{16}$.

\section{Methods}


150 Sequence data and inference of sex-linkage. RNA-seq data was generated in S. latifolia for a

151 cross (parents and progeny) for three tissues (seedlings, leaves and flower buds) and analysed

152 using the SEX-DETector pipeline ${ }^{24}$. RNA-seq data was also generated for two outgroup species

153 (S. viscosa and S. vulgaris). Reference mapping bias was corrected using the program GSNAP ${ }^{29}$.

154 Inferences of sex-linked contigs were validated using three sources of information (literature, a

155 genetic map and flow-sorted Y chromosome sequences). See Supplementary Text S2 for details.

156 Allelic expression levels. Contigwise autosomal, $\mathrm{X}, \mathrm{Y}, \mathrm{X}+\mathrm{X}$ and $\mathrm{X}+\mathrm{Y}$ normalised allelic

157 expression levels were computed by summing read numbers for each X-linked or Y-linked alleles

158 for filtered SNPs of the contigs (Supplementary Text S2) for each individual separately and then

159 normalised using the library size and the number of studied sex-linked SNPs in the contig:

160

$$
\mathrm{E}=\mathrm{r} /(\mathrm{n} * \mathrm{l})
$$

161 With $\mathrm{E}=$ normalised expression level for a given individual, $\mathrm{r}=$ sum of total read counts, $\mathrm{n}=$

162 number of studied SNPs, l = library size of the individual (number of mapped reads). Allelic

163 expression levels were then averaged among individuals for each contig. In order to make $S$.

164 latifolia expression levels comparable to S. viscosa and S. vulgaris, S. vicosa and S. vulgaris

165 expression levels were estimated using only the filtered SNP positions used in S. latifolia.

166 Normalised expression levels computed as explained in equation (1) in the two outgroups were

167 then averaged together for leaves and flower buds as expression levels are highly correlated $\left(\mathrm{R}^{2}\right.$

1680.7 and 0.5 for flower buds and leaves respectively and p-value $<2.10^{-6}$ in both cases). Averaging

169 expression levels between the two outgroups allows to get closer to the ancestral autosomal

170 expression level.

171 Sex-biased expression. Sex-biased contigs were inferred as in Zemp et al ${ }^{30}$. See Supplementary

172 Text S2 for more detail. 
173 Expression divergence between S. latifolia and the two outgroups at the contig level. The

174 normalised difference in allelic expression between S. latifolia and the two outgroups (hereafter

$175 \Delta$ ) was computed in order to study how sex chromosome expression levels evolved in S. latifolia

176 compared to autosomal expression levels in the two outroups: $\Delta$ is equal to zero if $S$. latifolia and

177 the outgroups have equal expression levels, $\Delta$ is positive if $S$. latifolia has higher expression

178 levels compared to the outgroups and $\Delta$ is negative otherwise:

$179 \Delta=($ S. latifolia expression level - outgroup expression level) / (outgroup expression $180 \quad$ level) (2)

181 Sex-linked contigs were grouped by categories of degeneration level using the average $\mathrm{Y}$ 182 over X expression ratio in males. 200 autosomal contigs were randomly selected in order to have 183 similar statistical power among gene categories. $\Delta$ values for each allele (maternal and paternal 184 in males and females) and each gene category were compared to zero using a Wilcoxon test. P185 values were corrected for multiple testing using a Benjamini and Hochberg correction. The 186 estimated median $\Delta$, confidence intervals and adjusted p-values were then used to plot Figure 1 187 and Supplementary Figures S2 to S7.

188 Expression differences between maternal and paternal alleles at the SNP level. Maternal and 189 paternal alleles expression were compared in S. latifolia for autosomal and sex-linked SNPs. In 190 order to deal with the difference in numbers of autosomal versus sex-linked contigs 191 (Supplementary Table S2), 200 autosomal contigs were randomly selected in order to keep 192 comparable powers of detection. Allelic expression levels in S. latifolia for each individual at 193 every SNP position were analysed using a linear regression model with mixed effects with the R 194 package lme4. We assumed a normal distribution of the read count data after log transformation.

195 In order to account for inter-individual and inter-contig variability, a random "individual" and a 
196 random "contig” effect were included in the model. The aim of this modeling framework was to

197 estimate the joint effect of the chromosomal origin of alleles (paternal or maternal in males or

198 females) and the status of the gene (autosomal or sex-linked with various levels of $\mathrm{Y}$

199 degeneration defined by the average $\mathrm{Y}$ over $\mathrm{X}$ expression ratio in males). Two fixed effects with

200 interaction were therefore considered in the model, see equation (3). In order to estimate the

201 changes in sex-linked gene expression levels since the evolution of sex chromosomes, we used

202 the average of the two outgroup expression levels as a reference (offset) for every SNP position,

203 divided by two in order to be comparable to S. latifolia allelic expression levels.

$204 \log ($ Expression +1$) \sim$ Chromosome $*$ Degeneration $+(1 \mid$ individual $)+(1 \mid$ Contig $)$, offset $=$

$205 \log ($ outgroup average expression/2 +1)

206 All effects of the model (fixed or random) were proved highly significant (p-values <

207 2.2.10 ${ }^{-16}$ ) using comparison of the fit of model (3) to simpler nested models (removing one effect

208 at a time in model (3)). In order to statistically test whether there was a difference between the

209 effects of paternal and maternal alleles in females in different degeneration categories we used

210 the contrasts provided by the lmerTest package in R. This strategy provided estimates,

211 confidence intervals and p-values of the difference between the two effects of paternal and

212 maternal origin in females in interaction with degeneration levels, while normalising by the

213 expression of the two outgroups. Moreover, the presence of random effects allows to account for

214 inter-individual and inter-contig variability. Finally, p-values were corrected for multiple testing

215 using a Benjamini and Hochberg correction. These values were used to plot Figure 2 and

216 Supplementary Figures S8 to S13. 
217 Data Availability. The new sequence data presented here can be downloaded from the European

218 Nucleotide Archive (ENA) under accession number PRJEB24933.

\section{References}

1. Bachtrog, D. Y-chromosome evolution: emerging insights into processes of Y-chromosome degeneration. Nat. Rev. Genet. 14, 113-124 (2013).

2. Charlesworth, B. The evolution of chromosomal sex determination and dosage compensation. Curr. Biol. CB 6, 149-162 (1996).

3. Mank, J. E. Sex chromosome dosage compensation: definitely not for everyone. Trends Genet. TIG 29, 677-683 (2013).

4. Gu, L. \& Walters, J. Sex chromosome dosage compensation evolution in animals: a beautiful theory, undermined by facts and bedeviled by details. Genome Biol. Evol. (2017).

5. Muyle, A., Shearn, R. \& Marais, G. A. The Evolution of Sex Chromosomes and Dosage Compensation in Plants. Genome Biol. Evol. 9, 627-645 (2017).

6. Kuroda, M. I., Hilfiker, A. \& Lucchesi, J. C. Dosage Compensation in Drosophila-a Model for the Coordinate Regulation of Transcription. Genetics 204, 435-450 (2016).

7. Albritton, S. E. et al. Sex-biased gene expression and evolution of the $\mathrm{x}$ chromosome in nematodes. Genetics 197, 865-883 (2014).

8. Ercan, S. Mechanisms of x chromosome dosage compensation. J. Genomics 3, 1-19 (2015).

9. Pessia, E., Engelstädter, J. \& Marais, G. A. B. The evolution of X chromosome inactivation in mammals: the demise of Ohno's hypothesis? Cell. Mol. Life Sci. CMLS 71, 1383-1394 (2014).

10. Disteche, C. M. Dosage compensation of the sex chromosomes. Annu. Rev. Genet. 46, $537-$ 560 (2012). 
11. Julien, P. et al. Mechanisms and evolutionary patterns of mammalian and avian dosage compensation. PLoS Biol. 10, e1001328 (2012).

12. Pessia, E., Makino, T., Bailly-Bechet, M., McLysaght, A. \& Marais, G. A. B. Mammalian X chromosome inactivation evolved as a dosage-compensation mechanism for dosage-sensitive genes on the X chromosome. Proc. Natl. Acad. Sci. U. S. A. 109, 5346-5351 (2012).

13. Whitworth, D. J. \& Pask, A. J. The X factor: X chromosome dosage compensation in the evolutionarily divergent monotremes and marsupials. Semin. Cell Dev. Biol. 56, 117-121 (2016).

14. Patten, M. M. et al. The evolution of genomic imprinting: theories, predictions and empirical tests. Heredity 113, 119-128 (2014).

15. Wagschal, A. \& Feil, R. Genomic imprinting in the placenta. Cytogenet. Genome Res. 113, 90-98 (2006).

16. Ohno, S. Sex chromosomes and sex-linked genes. Springer-Verl. Berl. Heidelb. N. Y. (1967).

17. Rautenberg, A., Hathaway, L., Oxelman, B. \& Prentice, H. C. Geographic and phylogenetic patterns in Silene section Melandrium (Caryophyllaceae) as inferred from chloroplast and nuclear DNA sequences. Mol. Phylogenet. Evol. 57, 978-991 (2010).

18. Chibalina, M. V. \& Filatov, D. A. Plant Y chromosome degeneration is retarded by haploid purifying selection. Curr. Biol. CB 21, 1475-1479 (2011).

19. Bergero, R. \& Charlesworth, D. Preservation of the Y transcriptome in a 10-million-year-old plant sex chromosome system. Curr. Biol. CB 21, 1470-1474 (2011).

20. Muyle, A. et al. Rapid de novo evolution of X chromosome dosage compensation in Silene latifolia, a plant with young sex chromosomes. PLoS Biol. 10, e1001308 (2012).

21. Kazama, Y. et al. SIWUS1; an X-linked gene having no homologous Y-linked copy in Silene latifolia. G3 Bethesda Md 2, 1269-1278 (2012).

22. Bergero, R., Qiu, S. \& Charlesworth, D. Gene Loss from a Plant Sex Chromosome System. Curr. Biol. CB (2015). doi:10.1016/j.cub.2015.03.015 
23. Papadopulos, A. S. T., Chester, M., Ridout, K. \& Filatov, D. A. Rapid Y degeneration and dosage compensation in plant sex chromosomes. Proc. Natl. Acad. Sci. U. S. A. 112, 1302113026 (2015).

24. Muyle, A. et al. SEX-DETector: A Probabilistic Approach to Study Sex Chromosomes in Non-Model Organisms. Genome Biol. Evol. 8, 2530-2543 (2016).

25. Ellegren, H. \& Parsch, J. The evolution of sex-biased genes and sex-biased gene expression. Nat. Rev. Genet. 8, 689-698 (2007).

26. Malone, J. H. et al. Mediation of Drosophila autosomal dosage effects and compensation by network interactions. Genome Biol. 13, r28 (2012).

27. Veitia, R. A., Veyrunes, F., Bottani, S. \& Birchler, J. A. X chromosome inactivation and active $\mathrm{X}$ upregulation in therian mammals: facts, questions, and hypotheses. J. Mol. Cell Biol. 7, 2-11 (2015).

28. Siroky, J., Castiglione, M. R. \& Vyskot, B. DNA methylation patterns of Melandrium album chromosomes. Chromosome Res. Int. J. Mol. Supramol. Evol. Asp. Chromosome Biol. 6, 441-446 (1998).

29. Wu, T. D. \& Nacu, S. Fast and SNP-tolerant detection of complex variants and splicing in short reads. Bioinforma. Oxf. Engl. 26, 873-881 (2010).

30. Zemp, N. et al. Evolution of sex-biased gene expression in a dioecious plant. Nat. Plants 2, 16168 (2016).

\section{Supplementary Materials:}

221 Supplementary Information includes Supplementary Texts S1-S2, Supplementary Figures S1222 S14 and Supplementary Tables S1-S3. 


\section{Acknowledgments}

224 This project was supported through an ANR grant ANR-14-CE19-0021-01 to G.A.B.M, SNSF 225 grant 160123 to A.W and Czech Science Agency grant number 16-08698S to R.H. We thank 226 Brandon Gaut, Tatiana Giraud and Judith Mank for comments on the manuscript.

\section{Author contributions}

228 Aline Muyle, Niklaus Zemp, Alex Widmer and Gabriel Marais conceived the study and 229 experimental design. Niklaus Zemp and Alex Widmer prepared and sequenced the plant material. 230 Aline Muyle ran SEX-DETector on the RNA-seq datasets for the three tissues, analysed the data, 231 prepared Tables and Figures and wrote the Supplementary Material with inputs from other 232 authors. Niklaus Zemp generated the X chromosome genetic map (with help from Aline Muyle 233 for the mapping and genotyping part). Radim Cegan, Jan Vrana and Roman Hobza did the Y 234 chromosome flow cytometry sorting and sequencing. Clothilde Deschamps did the first assembly 235 of the sorted Y chromosome and improved it with RNA-seq data with the help of Cecile 236 Fruchard. Aline Muyle did the blasts to validate the inferences of SEX-DETector. Raquel Tavares 237 did the GO term analysis. Aline Muyle and Frank Picard did the statistical analyses of the data. 238 Gabriel Marais and Aline Muyle wrote the main text of the manuscript with inputs from other 239 authors.

\section{Author information}

241 The authors declare no competing interests. 
242 Correspondence and requests for materials should be addressed to Aline Muyle (email:

243 muyle.aline@gmail.fr). 


\section{Figures}

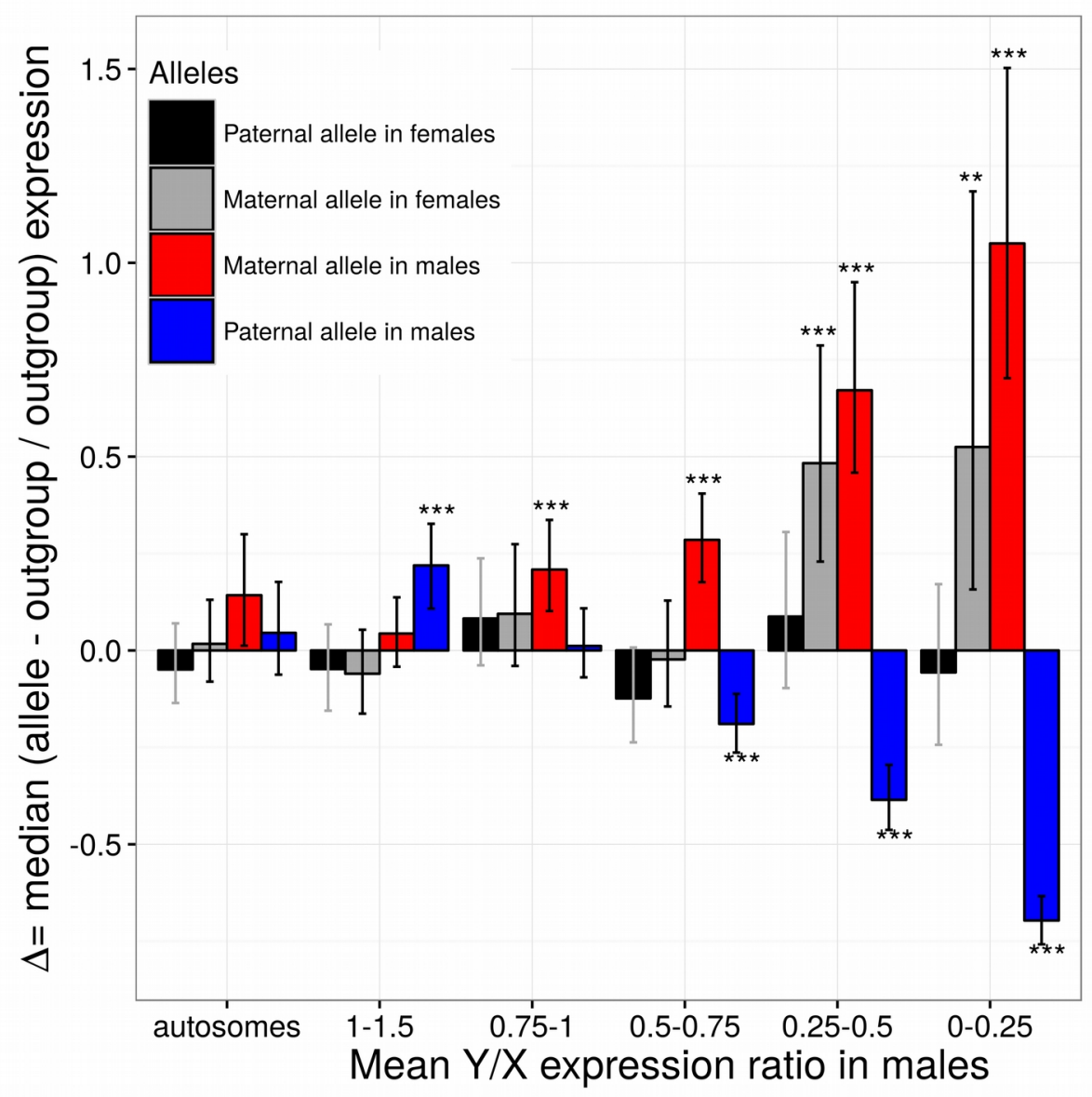

Figure 1: Normalised difference (hereafter $\Delta$ ) in allelic expression levels between S. latifolia and the outgroup without sex chromosomes $S$. vulgaris, in autosomal and sex-linked contigs for the seedling tissue. If $\Delta$ is lower, higher or equal to zero, then expression in $S$. latifolia is respectively lower, higher or equal to the outgroup (See Materials and test. The median $\Delta$, confidence intervals and p-values adjusted for multiple testing using a Benjamini and Hochberg correction are shown $\left({ }^{* * *}\right.$ : p-value $<0.001$; **: p-value < $0.01, *$ : p-value $<0.05)$. Allelic expression at SNP positions was averaged for each contig separately and the $\mathrm{Y} / \mathrm{X}$ ratio was used as a proxy for $\mathrm{Y}$ degeneration to group contigs. 
Contigs with sex-biased expression were removed, as well as contigs with $\mathrm{Y} / \mathrm{X}$ expression ratios above 1.5. Sample sizes for the different contig categories are: autosomal: 200; 1-1.5:148; $0.75-1: 139 ; 0.5-0.75: 160 ; 0.25-0.5: 114 ; 0-0.25: 79$ (we randomly selected 200 autosomal contigs to ensure similar statistical power among gene 258 categories).

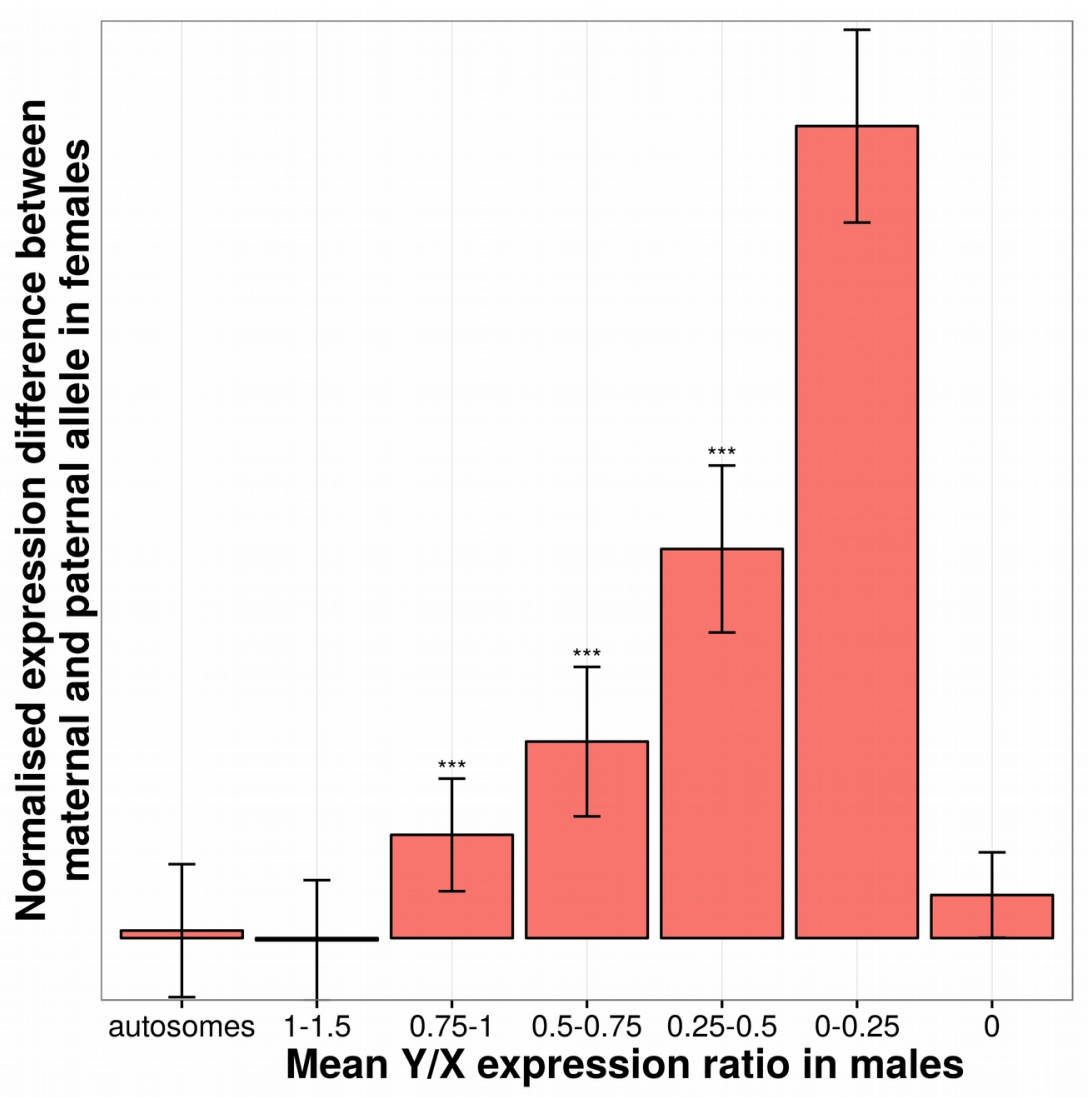

Figure 2: Normalised expression difference between maternal and paternal alleles in S. latifolia females in autosomal and sex-linked SNPs in the seedling tissue. The Y axis unit is the 
normalised allelic read count difference in log scale. A linear regression model with mixed effects was used to estimate the normalised difference between the effect of paternal and maternal origin of alleles in interaction with the contig status (autosomal or sex-linked with various levels of Y degeneration), while accounting for inter-contig and inter-individual variability (see Materials and Methods for details). The analysis is SNPwise and reveals consistent patterns across SNPs. See Fig. 1 legend for sample sizes for the different contig categories and statistical significance symbols.
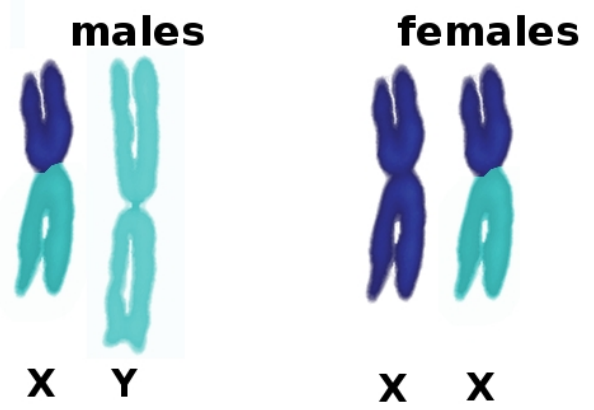

hypomethylated

DNA

hypermethylated

DNA

268 Figure 3: Illustration of DNA methylation staining results in S. latifolia from Siroky et al. ${ }^{28}$. See

269 Supplementary Figure S14 for the original Figure. One arm of one of the two X chromosomes in females was hypomethylated, as well as the same arm of the single $\mathrm{X}$ in males. 


\section{Supplementary Materials}

272 Supplementary Text S1: Dosage compensation in X-hemizygous 273 genes

The first papers on dosage compensation in S. latifolia were contradictory because they

275 focused on different gene sets. Muyle et al. ${ }^{1}$ focused on X/Y gene pairs while other papers

276 focused on X-hemizygous genes ${ }^{2,3}$. However, the X-hemizygous gene sets returned by the RNA-

277 seq approach used in those papers is less reliable than the $\mathrm{X} / \mathrm{Y}$ gene sets ${ }^{4}$. A gene might be

278 inferred as $\mathrm{X}$-hemizygous simply because the - still functional - Y copy is not expressed in the

279 tissue sampled for RNA-seq. In S. latifolia, X-hemizygous genes tend to be less expressed than

$280 \mathrm{X} / \mathrm{Y}$ genes and are less likely to be detected by segregation analysis as efficient SNP calling

281 requires a certain read depth, see ${ }^{4}$. Moreover, X-hemizygous genes are inferred from X

282 polymorphisms while $\mathrm{X} / \mathrm{Y}$ genes can be detected both with $\mathrm{X}$ and $\mathrm{X} / \mathrm{Y}$ polymorphisms, which

283 are more numerous. Another inherent bias to X-hemizygous contig inference comes from the

284 assembly step. If the $\mathrm{X}$ and the $\mathrm{Y}$ copy are too divergent to be assembled together, the $\mathrm{X}$ contig

285 will be wrongly inferred as $\mathrm{X}$-hemizygous because $\mathrm{Y}$ alleles will be absent from the contig (this

286 bias was at least partly corrected in the analyses presented here, see Material and Method section

287 5.1). The inferences of X-hemizygous genes using the RNA-seq approach (including SEX-

288 DETector) imply a higher rate of both false positives and false negatives than those for X/Y gene

289 pairs. In Papadopoulos et al. ${ }^{5}, 25 \%$ of the $\mathrm{X} / \mathrm{Y}$ chromosomes were sequenced using a genomic

290 approach. A much higher fraction of X-hemizygous genes was found than in previous RNA-seq

291 papers $^{2,3}$. Papadopoulos et al..$^{5}$ did find evidence for dosage compensation in approximately half 
292 of X-hemizygous genes (see their figure 3D). Due to limitations of the RNA-seq approach in

293 inferring X-hemizygous genes, results on X-hemizygous contigs are analysed separately here.

294 Poor dosage compensation of X-hemizygous contigs compared to X/Y contigs with high

295 Y degeneration was observed across all tissues (Supplementary Figures 2 to 7). Also, the parental

296 origin of the $\mathrm{X}$ chromosome has limited to no effect on female $\mathrm{X}$ expression levels for $\mathrm{X}$ -

297 hemizygous contigs, unlike X/Y contigs (Supplementary Figures 8 to 13). A reason that could

298 explain such a different pattern for $\mathrm{X}$-hemizygous genes compared to $\mathrm{X} / \mathrm{Y}$ genes is the possible

299 dosage insensitivity of X-hemizygous genes. X-hemizygous genes could have lost their Y copy

300 because dosage was not important for them and selection neither slowed down the loss of the $\mathrm{Y}$

301 copy nor selected for dosage compensation when degeneration inevitably occurred ${ }^{6}$. A well

302 described characteristic of dosage sensitive genes is that they tend to code proteins involved in

303 large complexes ${ }^{7}$. Gene Ontology was studied using the Blast2GO PRO version $2.7 .2^{30}$ as in ${ }^{8}$.

304 Using the GO-term analysis, our set of X-hemizygous contigs were found to be significantly

305 depleted in ribosomal protein coding genes compared to autosomal genes ( $\mathrm{p}$-value 1.3.10 ${ }^{-4}$ ),

306 which is consistent with the global dosage insensitivity of X-hemizygous genes in S. latifolia.

307 This depletion in large protein complexes was not found when comparing $\mathrm{X} / \mathrm{Y}$ genes to 308 autosomal genes.

Supplementary Text S2:

\section{1) RNA-seq Illumina data}


313 GSE35563, European Nucleotide Archive PRJEB14171), it included flower buds and leaf tissues

314 from individuals of a cross in S. latifolia as well as individuals in S. vulgaris. In addition to these

315 preexisting data, RNA-seq reads were generated in a comparable way for seedlings of a

316 controlled cross using the same parents in S. latifolia, four males and four females were sampled

317 (Seed_lati_female_1, Seed_lati_female_2, Seed_lati_female_3, Seed_lati_female_4,

318 Seed_lati_male_1, Seed_lati_male_2, Seed_lati_male_3 and Seed_lati_male_4). Seedlings were

319 also sequenced for S. vulgaris (Seed_vulg_herm_1, Seed_vulg_herm_2, Seed_vulg_herm_3 and

320 Seed_vulg_herm_4). Seedlings were grown in a temperature controlled climate chamber in

321 Eschikon (Switzerland) using the same conditions as in ${ }^{8}$. The S. latifolia and S. vulgaris

322 seedlings were collected without roots at the four-leaf stage. The sexing of the S. latifolia

323 seedlings was done using $\mathrm{Y}$ specific markers ${ }^{9}$ that were amplified with the direct PCAR

324 KAPA3G Plant PCR Kit (however male number 3 was later shown to be a female). High quality

325 RNA (RIN > 8.5) was extracted using the total RNA mini kit from Geneaid. Twelve RNA-seq

326 libraries were produced using the Truseq kit v2 from Illimina. Libraries were tagged individually

327 and sequenced in two Illumina Hiseq 2000 channels at the D-BSSE (ETH Zürich, Switzerland)

328 using 100 bp paired-end read protocol.

329 S. viscosa seeds we received from botanical gardens or collected in the wild by Bohuslav

330 Janousek and grown under controlled conditions in a greenhouse in Eschikon (Switzerland) and

331 Lyon (France). Similarly to ${ }^{8}$, flower buds after removing the calyx and leaves were collected.

332 Total RNA were extracted through the Spectrum Plant Total RNA kit (Sigma, Inc., USA)

333 following the manufacturer's protocol and treated with a DNAse. Libraries were prepared with

334 the TruSeq RNA sample Preparation v2 kit (Illumina Inc., USA). Each 2 nM cDNA library was 
335

336

337

338

339

340

341

342

343

344

345

346

347

348

349

350

351

352

353

354

355

356

357

sequenced using a paired-end protocol on a HiSeq2000 sequencer. Demultiplexing was performed using CASAVA 1.8.1 (Illumina) to produce paired sequence files containing reads for each sample in Illumina FASTQ format. RNA extraction and sequencing were done by the sequencing platform in the AGAP laboratory, Montpellier, France (http://umr-agap.cirad.fr/).

A female individual from an interspecific S. latifolia cross (C1_37) was back crossed with a male from an 11 generation inbred line (U10_49). The offspring (hereafter called BC1 individuals) were grown under controlled conditions in a greenhouse in Eschikon (Switzerland). High quality RNA from flower buds as described in ${ }^{10}$ was extracted from 48 BC1 individuals (35 females and 13 males). 48 RNA-seq libraries were produced using the Truseq kit v2 from Illimina with a median insert size of about 200 bp. Individuals were tagged separately and sequenced in four Illumna Hiseq 2000 channels at the D-BSSE (ETH Zürich, Switzerland) using 100bp paired-end read protocol. The parents used for this back cross had previously been sequenced in a similar way ${ }^{1,8}$.

\section{2) DNA-seq data from filtered $Y$ chromosome}

Y chromosome DNA was isolated using flow cytometry. The samples for flow cytometric experiments were prepared from root tips according to ${ }^{11}$ with modifications. Seeds of S. latifolia were germinated in a petri dish immersed in water at $25^{\circ} \mathrm{C}$ for 2 days until optimal length of roots was achieved $(1 \mathrm{~cm})$. The root cells were synchronized by treatment with $2 \mathrm{mM}$ hydroxyurea at $25^{\circ} \mathrm{C}$ for $18 \mathrm{~h}$. Accumulation of metaphases was achieved using $2.5 \mu \mathrm{M}$ oryzalin. Approximately 200 root tips were necessary to prepare $1 \mathrm{ml}$ of sample. The chromosomes were released from the root tips by mechanical homogenization using a Polytron PT1200 homogenizer (Kinematica AG, Littau, Switzerland) at 18,000rpm for $13 \mathrm{~s}$. The crude suspension was filtered and stained with DAPI $(2 \mu \mathrm{g} / \mathrm{ml})$. All flow cytometric experiments were performed on FACSAria 
II SORP flow cytometer (BD Biosciences, San José, Calif., USA). Isolated Y chromosomes were

359 sequenced with 2x100bp PE Illumina HiSeq.

\section{3) RNA-seq PacBio data}

361

Plants from an 11 generation inbred line were grown under controlled conditions in a 362 greenhouse in Eschikon (Switzerland). One male (U11_02) was randomly selected. High quality RNA (RIN > 7.5) were extracted using the total RNA mini kit of Geneaid from very small flower buds, small and large flower buds, flowers before anthesis without calyces, rosette leaves, seedlings (4 leaves stage) and pollen. RNA of the different tissues was equally pooled and cDNA was produced using the Clontech SMARTer Kit. The cDNA pool was then normalized using a

$\mathrm{kb}$ and $1.2-2 \mathrm{~kb}$ ) using the Pippin Prep (Sage Science). Two SMRTbell libraries were prepared on a PacBio RS II at the Functional Genomic Center Zurich (FGCZ).

371

372

\section{4) RNA-seq 454 data}

Previously generated 454 data was used ${ }^{8,12}$.

\section{2) Reference trancriptome assembly}

The same reference transcriptome as in Muyle et al. ${ }^{12}$ and Zemp et al. ${ }^{8}$ was used.

\section{3) Inference of sex-linked contigs}

Autosomal and sex-linked contigs were inferred as in Muyle et al. ${ }^{12}$ and Zemp et al. ${ }^{8}$. Illumina reads from the individuals of the cross were mapped onto the assembly using BWA ${ }^{13}$ version 0.6.2 with the following parameters: bwa aln -n 5 and bwa sampe. The libraries were then merged using SAMTOOLS version $0.1 .18^{14}$. The obtained alignments were locally 
realigned using GATK IndelRealigner ${ }^{15}$ and were analysed using reads2snps ${ }^{16}$ version 3.0 with

381 the following parameters: -fis 0 -model M2 -output_genotype best -multi_alleles acc

382 -min_coverage 3 -par false. This allowed to genotype individuals at each loci while allowing for

383 biases in allele expression, and without cleaning for paralogous SNPs. Indeed, X/Y SNPs tend to

384 be filtered out by paraclean, a program which removes paralogous positions ${ }^{17}$. A second run of

385 genotyping was done with paraclean in order to later remove paralogous SNPs from autosomal

386 contigs only. SEX-DETector ${ }^{12}$ was then used to infer contig segregation types after estimation of

387 parameters using an SEM algorithm. Contig posterior segregation type probabilities were filtered

388 to be higher than 0.8 . Because the parents were not sequenced for the leaf and seedling datasets,

389 SEX-DETector was run using the flower bud data for the parents.

\section{4) Reference mapping bias correction}

In order to avoid biases towards the reference allele in expression level estimates, a second mapping was done using the program $\mathrm{GSNAP}^{18}$ with SNP tolerant mapping option. A GSNAP SNP file was generated by home-made perl scripts using the SEX-DETector SNP detail output file. Shortly, for each polymorphic position of all contigs, the most probable posterior SNP type was used to extract the possible alleles and write them to the GSNAP SNP file. This way, reference mapping bias was corrected for both sex-linked and autosomal contigs. Only uniquely mapped and concordant paired reads were kept after this. See Supplementary Table S1 for percentage of mapped reads. SEX-DETector was run a second time on this new mapping and the new inferences were used afterwards for all analyses (see Supplementary Table S2 for 400 inference results).

\section{1) Detection of false $X$-hemizygous contigs}


$404 \mathrm{Y}$ copies were assembled into different contigs. In order to detect such cases, $\mathrm{X}$-hemizygous 405 contigs were blasted ${ }^{19}$ with parameter -e $1 \mathrm{E}-5$ against RNA-seq contigs that have male-limited 406 expression (see section 7 below for how male-limited contigs were inferred). These cases were 407 removed from the analyses presented here.

\section{2) Validation using data from literature}

A few sex-linked and autosomal genes in S. latifolia have already been described in the literature (see Supplementary Table S3).

\section{3) Validation using a genetic map}

A genetic map was built and contigs from the $\mathrm{X}$ linkage group were used to validate

414 (hereafter CP) and backcross (hereafter BC1) were mapped against the reference transcriptome using $\mathrm{BWA}^{13}$ with a maximum number of mismatch equal to 5 . Libraries were merged and realigned using GATK ${ }^{15}$ and SNPs were analysed using reads2snps ${ }^{16}$. Using a customized perl script, SNP genotypes from the parents and the offspring as well as the associated posterior 418 probabilities were extracted from the reads2snps output file. Only SNPs with a reads2snps posterior genotyping probability higher than 0.8 were kept for further analyses. Then, only informative SNPs were kept: both parents had to be homozygous and different between father LEUK144-3 and mother U10_37 in a first generation backcross population design (BC1) and at least one allele had to be different between mother C1_37 and father U10_49 in the cross-

423 pollinator (CP). Filtered SNPs were then converted into a JoinMap format using a customized R script. If more than one informative SNP per contig was present, the SNP was used with less segregation distortion and less missing values. This led to 8,023 BC1 and 16,243 CP markers. 
Loci with more than $10 \%$ missing values were excluded, resulting in 7,951 BC1 and 15,118 CP

427 markers. Linkage groups were identified using the default setting of JoinMap $4.1^{20}$. Robustness

428 of the assignment of the linkage groups was tested using LepMap ${ }^{21}$. Blasting the contigs against

429 known sex-linked genes allowed the identification of the X chromosome linkage group. Contigs

430 could not be ordered along the linkage groups due to the too limited number of individuals that

431 prevented the convergence of contig order. However, contigs were reliably attributed to linkage 432 groups.

\section{4) Validation using isolated $Y$ chromosome DNA-seq data}

Filtered Y chromosome DNA-seq reads were filtered for quality and Illumina adapters

were removed using the ea-utils FASTQ processing utilities ${ }^{22}$. The optimal kmer value for assembly was searched using KmerGenie ${ }^{23}$. Filtered reads were assembled using soapdenovo $2^{24}$ with kmer=49, as suggested by KmerGenie. The obtained assembly was highly fragmented, therefore RNA-seq data was used to join, order and orient the genomic fragments with L_RNA_scaffolder ${ }^{25}$. The following RNA-seq reads were used (see section 1): one sample of male flower buds sequenced by 454, 6 samples of male flower buds sequenced by Illumina paired-end, 4 samples of male leaves sequenced by Illumina paired-end and one sample of male pooled tissues sequenced by PacBio. The genomic assembly was successively scaffolded with L_RNA_scaffolder using RNA-seq samples one after the other, first 454 samples then Illumina and finally PacBio. The obtained contigs were filtered to be longer than 200pb.

\section{5) Set of validated sex-linked and autosomal contigs}

The three sources of data (litterature, genetic map and filtered Y sequence data) were

-e 1E-5. Blasts were filtered for having a percentage of identity over $90 \%$, an alignment length 
over 100bp and were manually checked. If a sex-linked RNA-seq contig blasted against a

450 sequence from one of the three data sources (literature, X genetic map or filtered Y DNA-seq) it

451 was then considered as validated. See Supplementary Table S2 for numbers of validated sex-

452 linked contigs.

\section{6) Expression level estimates}

\section{1) whole contig expression levels}

Whole contig mean expression levels were obtained for each individual using GATK

DepthOfCoverage ${ }^{15}$ as the sum of every position coverage, divided by the length of the contig.

457

460

461

462

463

464

465

466

467

468

469

Normalised expression levels, in $\mathrm{RPKM}^{26}$, were then computed for each individual by dividing

by the value by the library size of the individual (total number of mapped reads), accounting for different depths of coverage among individuals. Whole contig mean male and female expression levels were then computed by averaging male and female individuals for each contig.

\section{2) Allelic expression levels filtering}

In order to study separately $\mathrm{X}$ and $\mathrm{Y}$ allele expression levels in males and females, expression levels were studied at the SNP level. In S. latifolia, for each sex-linked contig expression levels were estimated using read counts from both $\mathrm{X} / \mathrm{Y}$ and $\mathrm{X}$-hemizygous informative SNPs. SNPs were attributed to an $\mathrm{X} / \mathrm{Y}$ or $\mathrm{X}$ hemizygous segregation type if the according posterior probability was higher than 0.5. SNPs are considered informative if the father is heterozygous and has a genotype that is different from the mother (otherwise it is not possible to tell apart the $\mathrm{X}$ from the $\mathrm{Y}$ allele and therefore it is not possible to compute $\mathrm{X}$ and $\mathrm{Y}$ expression separately). X/Y SNPs for which at least one female had over two percent of her 
470

471 autosomal SNPs from autosomal contigs were used in a similar way.
472

473 Xs from the mother), Y expression level is only computed from the father as all males are

474 homozygous in the progeny. Such contigs were therefore removed when having under $3 \mathrm{X} / \mathrm{X}$

475 SNPs to avoid approximations on the contig mean Y/X expression level (39 contigs removed in

476 the flower buds dataset, 44 in the leaves dataset and 40 in the seedlings dataset).

477

478

480

481

482

483

484

485

486

487

488

489

490

In order to make S. latifolia expression levels comparable to S. viscosa and S. vulgaris for sex-linked contigs, S. vicosa and S. vulgaris expression levels were estimated using only the positions used in S. latifolia (informative X/Y or X-hemizygous SNPs). The read count of every position in every contig and for every S. viscosa and S. vulgaris individual was given by GATK DepthOfCoverage $^{15}$. Only positions corresponding to informative autosomal, $\mathrm{X} / \mathrm{Y}$ or $\mathrm{X}$ hemizygous SNPs in S. latifolia were used to compute the expression level for each contig and each individual as explained in equation (1).

Contigwise S. latifolia autosomal, $\mathrm{X}, \mathrm{Y}, \mathrm{X}+\mathrm{X}, \mathrm{X}+\mathrm{Y}$ allelic expression levels were then averaged among individuals. Autosomal normalised expression levels in the two outgroups ( $S$. vulgaris and S. viscosa) were averaged together.

\section{7) Identification of contigs with sex-biased expression}

The analysis was done separately for the three tissues (flower buds, seedling and rosette leaves) as in Zemp et al. ${ }^{8}$ using the $\mathrm{R}$ package edge ${ }^{27}$. See Supplementary Table S2 for number of sex-biased contigs removed in order to study dosage compensation. Male-limited expressed 
491 contigs were identified by calculating the mean expression values (FPKM) in both sexes and

492 selecting those which were exclusively expressed in males.

\section{$493 \quad$ Supplementary References}

1. Muyle, A. et al. Rapid de novo evolution of X chromosome dosage compensation in Silene latifolia, a plant with young sex chromosomes. PLoS Biol. 10, e1001308 (2012).

2. Chibalina, M. V. \& Filatov, D. A. Plant Y chromosome degeneration is retarded by haploid purifying selection. Curr. Biol. CB 21, 1475-1479 (2011).

3. Bergero, R., Qiu, S. \& Charlesworth, D. Gene Loss from a Plant Sex Chromosome System. Curr. Biol. CB (2015). doi:10.1016/j.cub.2015.03.015

4. Blavet, N. et al. Identifying new sex-linked genes through BAC sequencing in the dioecious plant Silene latifolia. BMC Genomics (2015).

5. Papadopulos, A. S. T., Chester, M., Ridout, K. \& Filatov, D. A. Rapid Y degeneration and dosage compensation in plant sex chromosomes. Proc. Natl. Acad. Sci. U. S. A. 112, 1302113026 (2015).

6. Muyle, A., Shearn, R. \& Marais, G. A. The Evolution of Sex Chromosomes and Dosage Compensation in Plants. Genome Biol. Evol. 9, 627-645 (2017).

7. Pessia, E., Makino, T., Bailly-Bechet, M., McLysaght, A. \& Marais, G. A. B. Mammalian X chromosome inactivation evolved as a dosage-compensation mechanism for dosage-sensitive genes on the X chromosome. Proc. Natl. Acad. Sci. U. S. A. 109, 5346-5351 (2012).

8. Zemp, N. et al. Evolution of sex-biased gene expression in a dioecious plant. Nat. Plants 2, 16168 (2016).

9. Hobza, R. \& Widmer, A. Efficient molecular sexing in dioecious Silene latifolia and S. dioica and paternity analysis in F(1) hybrids. Mol. Ecol. Resour. 8, 1274-1276 (2008). 
10. Zemp, N., Minder, A. \& Widmer, A. Identification of internal reference genes for gene expression normalization between the two sexes in dioecious white Campion. PloS One 9, e92893 (2014).

11. Vrána, J., Simková, H., Kubaláková, M., Cíhalíková, J. \& Doležel, J. Flow cytometric chromosome sorting in plants: the next generation. Methods San Diego Calif 57, 331-337 (2012).

12. Muyle, A. et al. SEX-DETector: A Probabilistic Approach to Study Sex Chromosomes in Non-Model Organisms. Genome Biol. Evol. 8, 2530-2543 (2016).

13. Li, H. \& Durbin, R. Fast and accurate short read alignment with Burrows-Wheeler transform. Bioinforma. Oxf. Engl. 25, 1754-1760 (2009).

14. Li, H. et al. The Sequence Alignment/Map format and SAMtools. Bioinforma. Oxf. Engl. 25, 2078-2079 (2009).

15. DePristo, M. A. et al. A framework for variation discovery and genotyping using nextgeneration DNA sequencing data. Nat. Genet. 43, 491-498 (2011).

16. Tsagkogeorga, G., Cahais, V. \& Galtier, N. The population genomics of a fast evolver: high levels of diversity, functional constraint, and molecular adaptation in the tunicate Ciona intestinalis. Genome Biol. Evol. 4, 740-749 (2012).

17. Gayral, P. et al. Reference-free population genomics from next-generation transcriptome data and the vertebrate-invertebrate gap. PLoS Genet. 9, e1003457 (2013).

18. Wu, T. D. \& Nacu, S. Fast and SNP-tolerant detection of complex variants and splicing in short reads. Bioinforma. Oxf. Engl. 26, 873-881 (2010).

19. Altschul, S. F., Gish, W., Miller, W., Myers, E. W. \& Lipman, D. J. Basic local alignment search tool. J. Mol. Biol. 215, 403-410 (1990).

20. VAN Ooijen, J. W. Multipoint maximum likelihood mapping in a full-sib family of an outbreeding species. Genet. Res. 93, 343-349 (2011). 
21. Rastas, P., Paulin, L., Hanski, I., Lehtonen, R. \& Auvinen, P. Lep-MAP: fast and accurate linkage map construction for large SNP datasets. Bioinforma. Oxf. Engl. 29, 3128-3134 (2013).

22. Aronesty, E. ea-utils: 'Command-line tools for processing biological sequencing data'. (2011).

23. Chikhi, R. \& Medvedev, P. Informed and automated k-mer size selection for genome assembly. Bioinforma. Oxf. Engl. 30, 31-37 (2014).

24. Luo, R. et al. SOAPdenovo2: an empirically improved memory-efficient short-read de novo assembler. GigaScience 1, 18 (2012).

25. Xue, W. et al. L_RNA_scaffolder: scaffolding genomes with transcripts. BMC Genomics 14, 604 (2013).

26. Oshlack, A., Robinson, M. D. \& Young, M. D. From RNA-seq reads to differential expression results. Genome Biol. 11, 220 (2010).

27. Robinson, M. D., McCarthy, D. J. \& Smyth, G. K. edgeR: a Bioconductor package for differential expression analysis of digital gene expression data. Bioinforma. Oxf. Engl. 26, 139-140 (2010).

28. R Development Core Team. R: A language and environment for statistical computing, reference index version 2.15.0. $R$ Foundation for Statistical Computing, Vienna, Austria (2012).

29. Benjamini, Y. \& Hochberg, Y. Controlling the False Discovery Rate: A Practical and Powerful Approach to Multiple Testing. J. R. Stat. Soc. Ser. B Methodol. 57, 289-300 (1995).

30. Conesa, A. et al. Blast2GO: a universal tool for annotation, visualization and analysis in functional genomics research. Bioinforma. Oxf. Engl. 21, 3674-3676 (2005).

31. Rautenberg, A., Hathaway, L., Oxelman, B. \& Prentice, H. C. Geographic and phylogenetic patterns in Silene section Melandrium (Caryophyllaceae) as inferred from chloroplast and nuclear DNA sequences. Mol. Phylogenet. Evol. 57, 978-991 (2010). 
32. Jenkins, C. \& Keller, S. R. A phylogenetic comparative study of preadaptation for invasiveness in the genus Silene (Caryophyllaceae). Biol. Invasions 13, 1471-1486 (2010).

33. Rautenberg, A., Sloan, D. B., Aldén, V. \& Oxelman, B. Phylogenetic Relationships of Silene multinervia and Silene Section Conoimorpha (Caryophyllaceae). Syst. Bot. 37, 226-237 (2012).

34. Siroky, J., Castiglione, M. R. \& Vyskot, B. DNA methylation patterns of Melandrium album chromosomes. Chromosome Res. Int. J. Mol. Supramol. Evol. Asp. Chromosome Biol. 6, 441-446 (1998).

\section{Supplementary Figures}

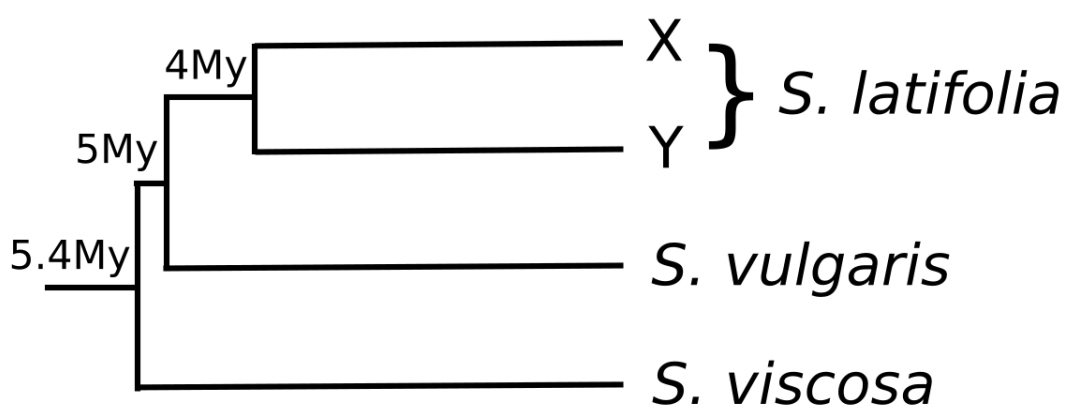

495 Supplementary Figure S1: Relatedness among the three studied species, extracted from ${ }^{31}$ ages

496 at the nodes are shown in million years (My). The exact relationship among species is poorly 497 resolved ${ }^{31-33}$. In some phylogenies $S$. viscosa is closest to S. latifolia, whereas in others $S$. 498 vulgaris is closest as shown here, and in others both species are equally diverged to S. latifolia. 


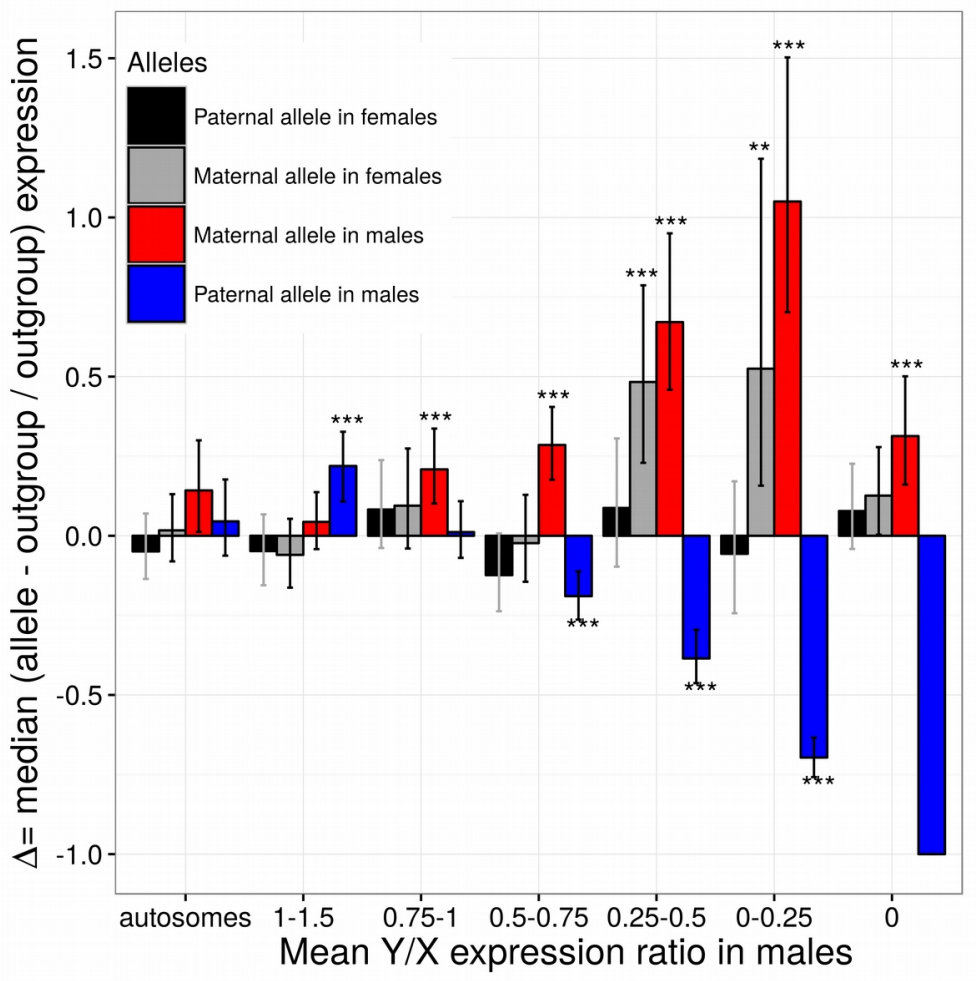

499 Supplementary Figure S2: Normalised difference in allelic expression levels between $S$.

500 latifolia and the two outgroups without sex chromosomes S. vulgaris and S. viscosa (hereafter

$501 \Delta$ ), in autosomal and sex-linked contigs for the seedling tissue. Maternal and paternal allelic read 502 numbers were summed at SNP positions and normalised for each individual separately, then 503 averaged among individuals for each contig. $\Delta$ was computed as follows: $\Delta=$ (allelic expression 504 in S. latifolia - allelic expression in the outgroup) / allelic expression in the outgroup). If $\Delta$ is 505 lower, higher or equal to zero, then expression in S. latifolia is respectively lower, higher or 506 equal to the outgroup. For all contig categories, $\Delta$ was compared to zero using a Wilcoxon test. 507 The median $\Delta$, confidence intervals and p-values adjusted for multiple testing using a Benjamini 508 and Hochberg correction are shown (***: p-value $<0.001 ; * *$ : p-value $<0.01$, *: p-value $<0.05)$.

509 The Y/X ratio was computed in S. latifolia males and averaged among individuals to use as a 
510 proxy for $\mathrm{Y}$ degeneration. $\mathrm{X}$-hemizygous contigs have a $\mathrm{Y} / \mathrm{X}$ ratio equal to zero. Contigs with 511 sex-biased expression were removed, as well as contigs with $\mathrm{Y} / \mathrm{X}$ expression ratios above 1.5.

512 Sample sizes for the different contig categories are: autosomal:200; 1-1.5:148; 0.75-1:139; 0.5-

$5130.75: 160 ; 0.25-0.5: 114 ; 0-0.25: 79 ; 0: 205$ (note that 200 autosomal contigs were randomly

514 selected in order to have similar statistical power among gene categories). In the absence of

515 dosage compensation, the single $\mathrm{X}$ in males should be expressed at levels similar to the outgroup

516 that does not have sex chromosomes, in other words, without dosage compensation $\Delta$ should be

517 close to zero for the maternal allele in males (red bars). Results show that the maternal allele is

518 hyper-expressed in S. latifolia when the $\mathrm{Y}$ chromosome is degenerated, both in males and 519 females. 


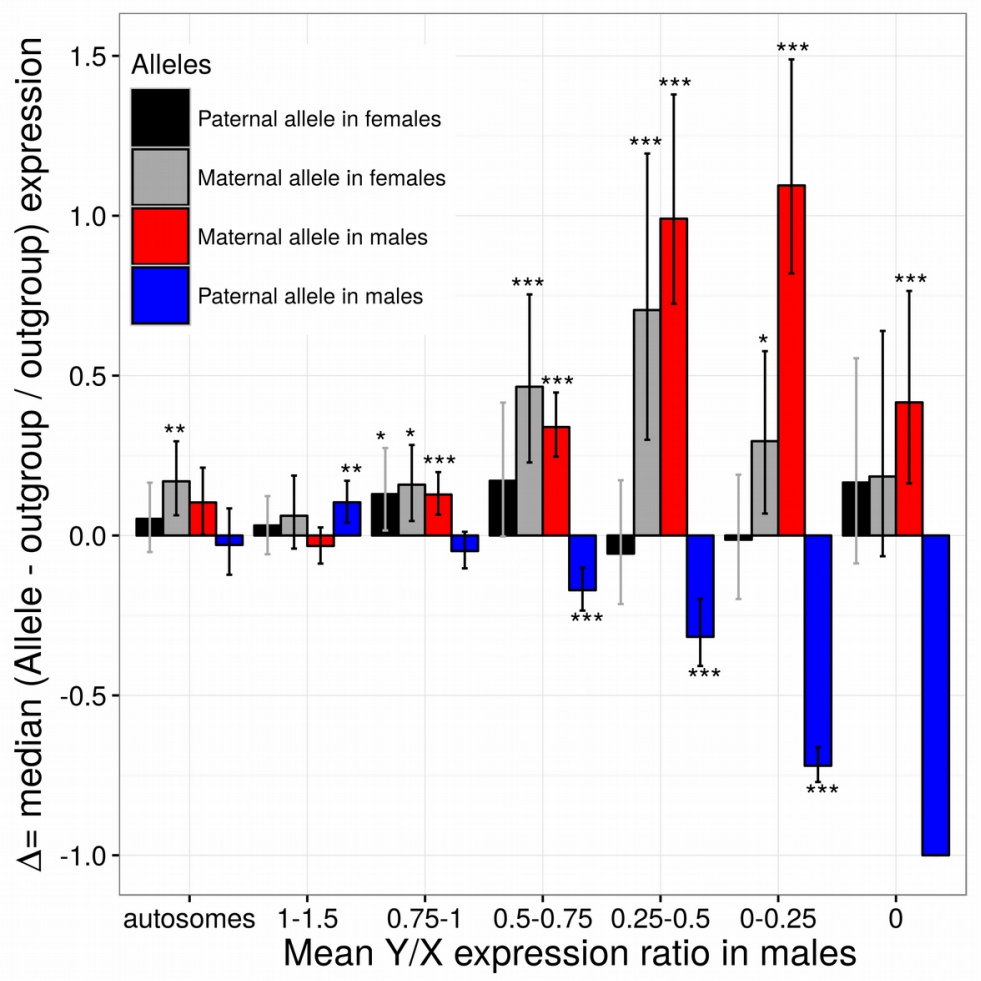

520 Supplementary Figure S3: Normalised difference in allelic expression levels between $S$.

521 latifolia and the two outgroups without sex chromosomes $S$. vulgaris and $S$. viscosa $(\Delta)$, in

522 autosomal and sex-linked contigs for the flower bud tissue. Same legend as Supplementary

523 Figure S2 except for sample sizes for the different contig categories: autosomal:200; 1-1.5:95;

$524 \quad 0.75-1: 195 ; 0.5-0.75: 203 ; 0.25-0.5: 176 ; 0-0.25: 116 ; 0: 103$. 


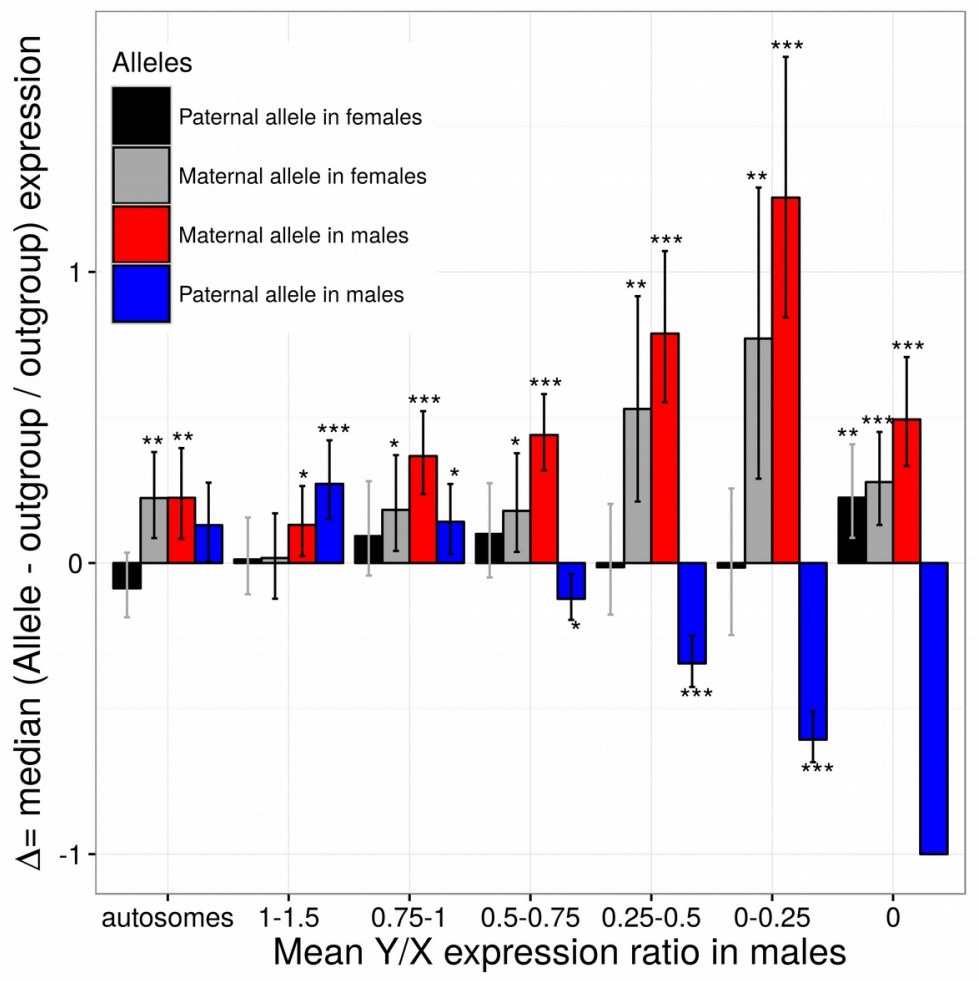

525 Supplementary Figure S4: Normalised difference in allelic expression levels between $S$.

526 latifolia and the two outgroups without sex chromosomes $S$. vulgaris and $S$. viscosa $(\Delta)$, in 527 autosomal and sex-linked contigs for the leaf tissue. Same legend as Supplementary Figure S2

528 except for sample sizes for the different contig categories: autosomal:200; 1-1.5:159; 0.75-1:132;

$529 \quad 0.5-0.75: 147 ; 0.25-0.5: 126 ; 0-0.25: 71 ; 0: 275$. 


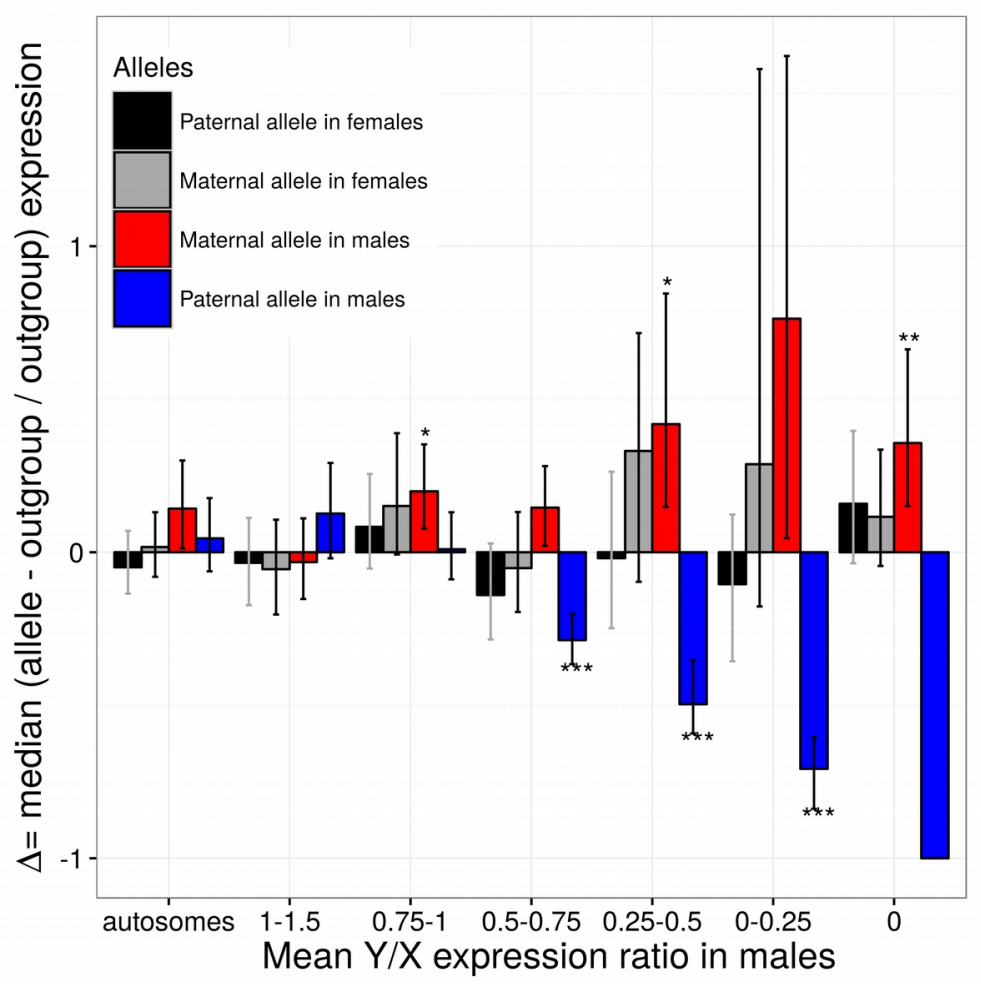

530 Supplementary Figure S5: Normalised difference in allelic expression levels between $S$. 531 latifolia and the two outgroups without sex chromosomes $S$. vulgaris and $S$. viscosa $(\Delta)$, in 532 autosomal and sex-linked contigs that were validated (see Materials and Methods), for the 533 seedling tissue. Same legend as Supplementary Figure S2 except for sample sizes for the 534 different contig categories: autosomal:77; 1-1.5:71; 0.75-1:82; 0.5-0.75:91; 0.25-0.5:44; 0535 0.25:29; 0:89. 


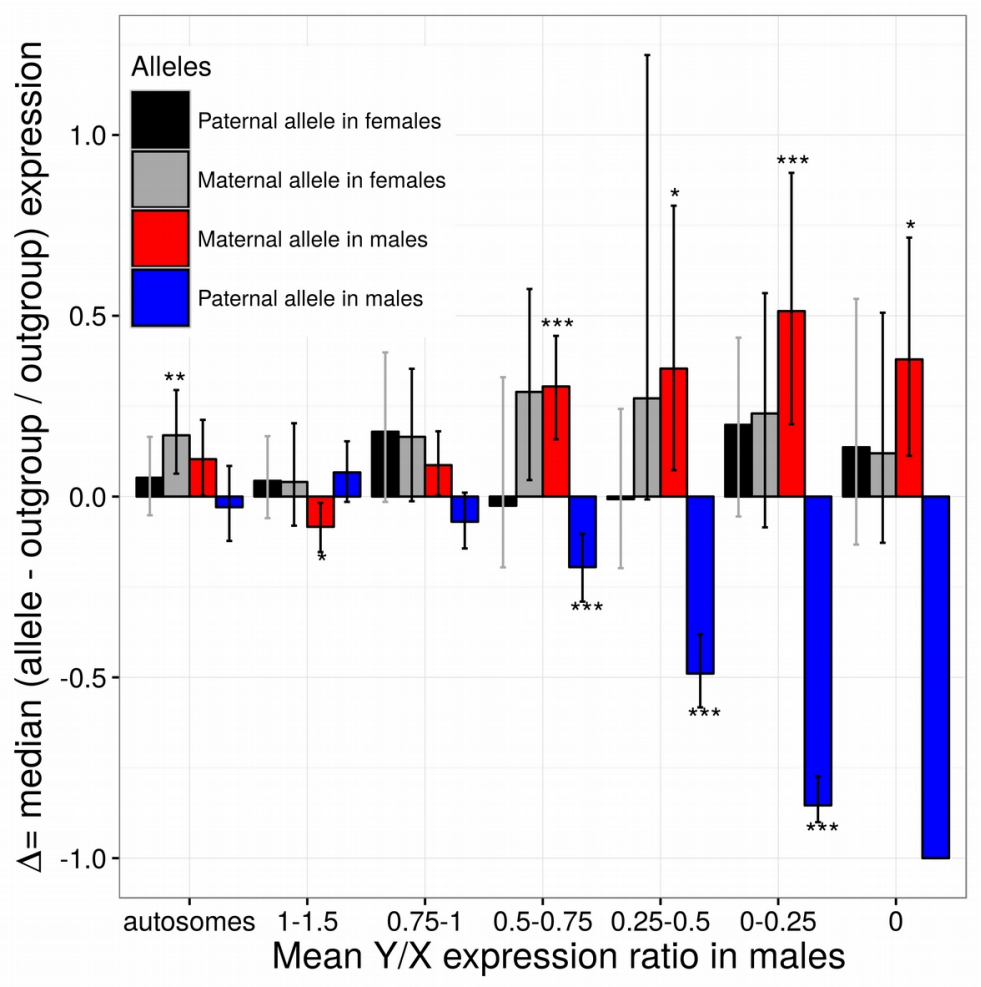

536 Supplementary Figure S6: Normalised difference in allelic expression levels between $S$.

537 latifolia and the two outgroups without sex chromosomes $S$. vulgaris and $S$. viscosa $(\Delta)$, in

538 autosomal and sex-linked contigs that were validated (see Materials and Methods), for the

539 flower bud tissue. Same legend as Supplementary Figure S2 except for sample sizes for the

540 different contig categories: autosomal:74; 1-1.5:86; 0.75-1:91; 0.5-0.75:67; 0.25-0.5:45; 0-

$541 \quad 0.25: 31 ; 0: 55$ 


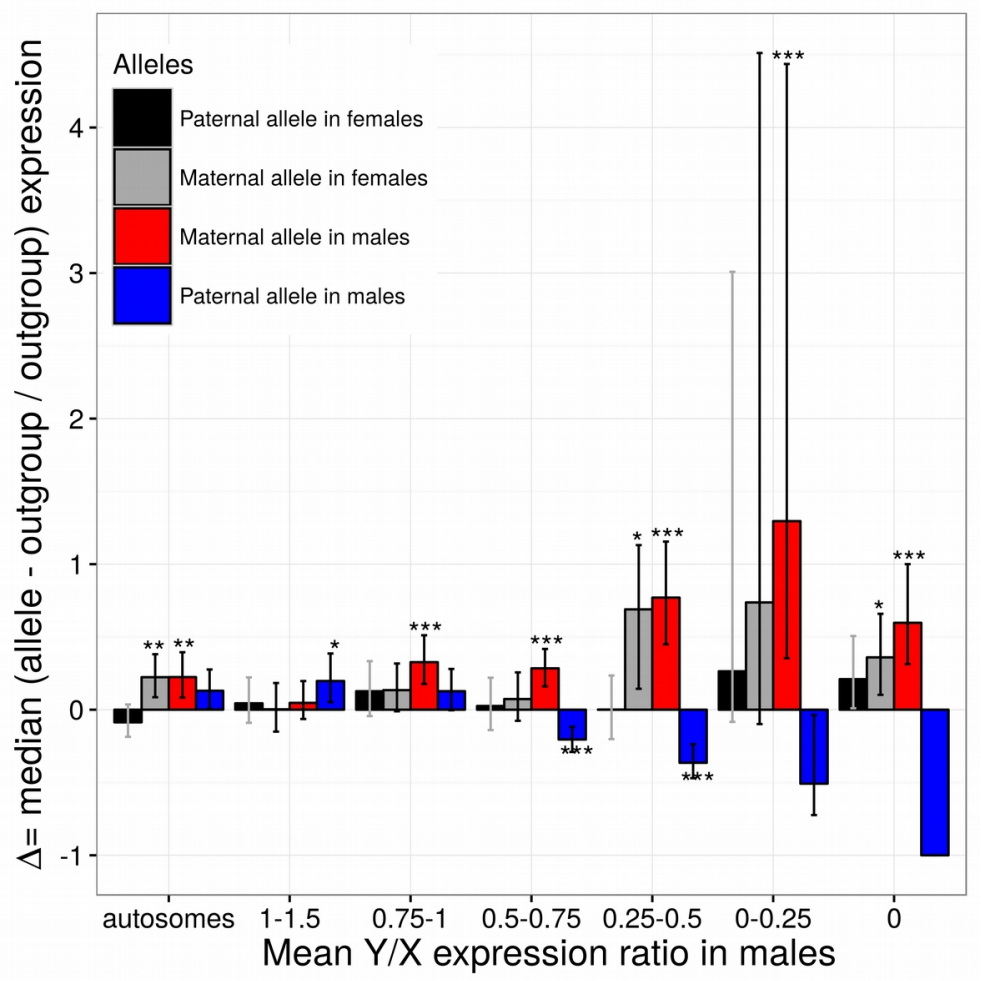

542 Supplementary Figure S7: Normalised difference in allelic expression levels between $S$.

543 latifolia and the two outgroups without sex chromosomes $S$. vulgaris and $S$. viscosa $(\Delta)$, in

544 autosomal and sex-linked contigs that were validated (see Materials and Methods), for the leaf

545 tissue. Same legend as Supplementary Figure S2 except for sample sizes for the different contig

546 categories: autosomal:79; 1-1.5:84; 0.75-1:74; 0.5-0.75:77; 0.25-0.5:52; 0-0.25:19; 0:119. 


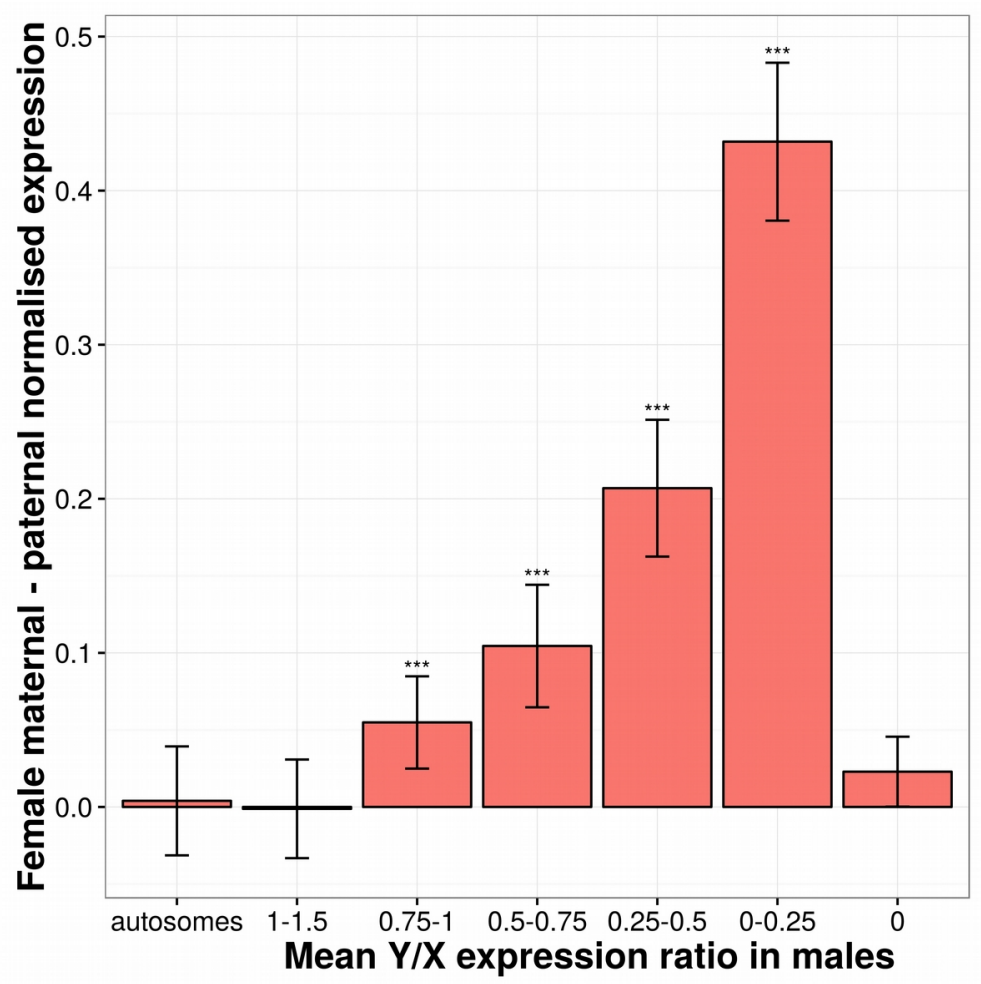

547 Supplementary Figure S8: Normalised expression difference between the maternal and paternal

548 allele in S. latifolia females in autosomal and sex-linked contigs for the seedling tissue. The $\mathrm{Y}$

549 axis unit is the normalised allelic read count difference in log scale. A linear regression model

550 with mixed effects was used to study allelic expression in S. latifolia for every SNP position. In

551 order to measure the changes in S. latifolia expression due to sex chromosomes evolution, the

552 outgroup $S$. vulgaris that does not have sex chromosomes was used as a reference in the model

553 (see Materials and Methods for details). The framework provided estimates for the normalised

554 difference between the effect of paternal and maternal origin of alleles in interaction with the

555 contig status (autosomal or sex-linked with various levels of Y degeneration), while accounting

556 for inter-contig and inter-individual variability. See Supplementary Figure S2 legend for sample

557 sizes for the different contig categories and statistical significance symbols. Results show that $\mathrm{Y}$

558 degeneration is linked to a significant expression difference between the paternal and maternal

559 alleles in females, which is not observed in autosomal and non-degenerated sex-linked contigs. 


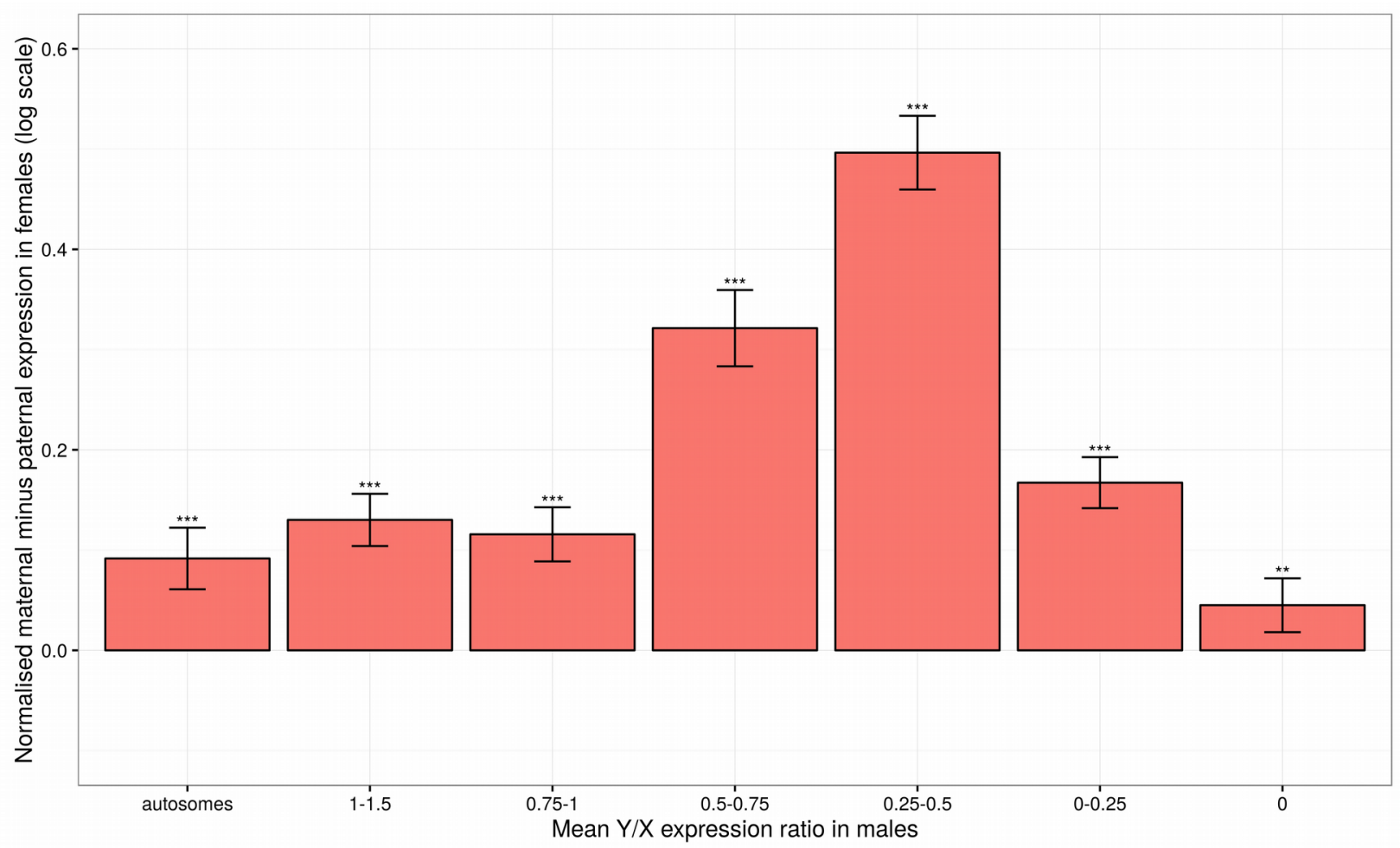

560 Supplementary Figure S9: Normalised expression difference between the maternal and paternal

561 allele in S. latifolia females in autosomal and sex-linked contigs for the flower bud tissue. See

562 supplementary Figure S8 for legend and Supplementary Figure S3 for sample sizes for the

563 different contig categories. 


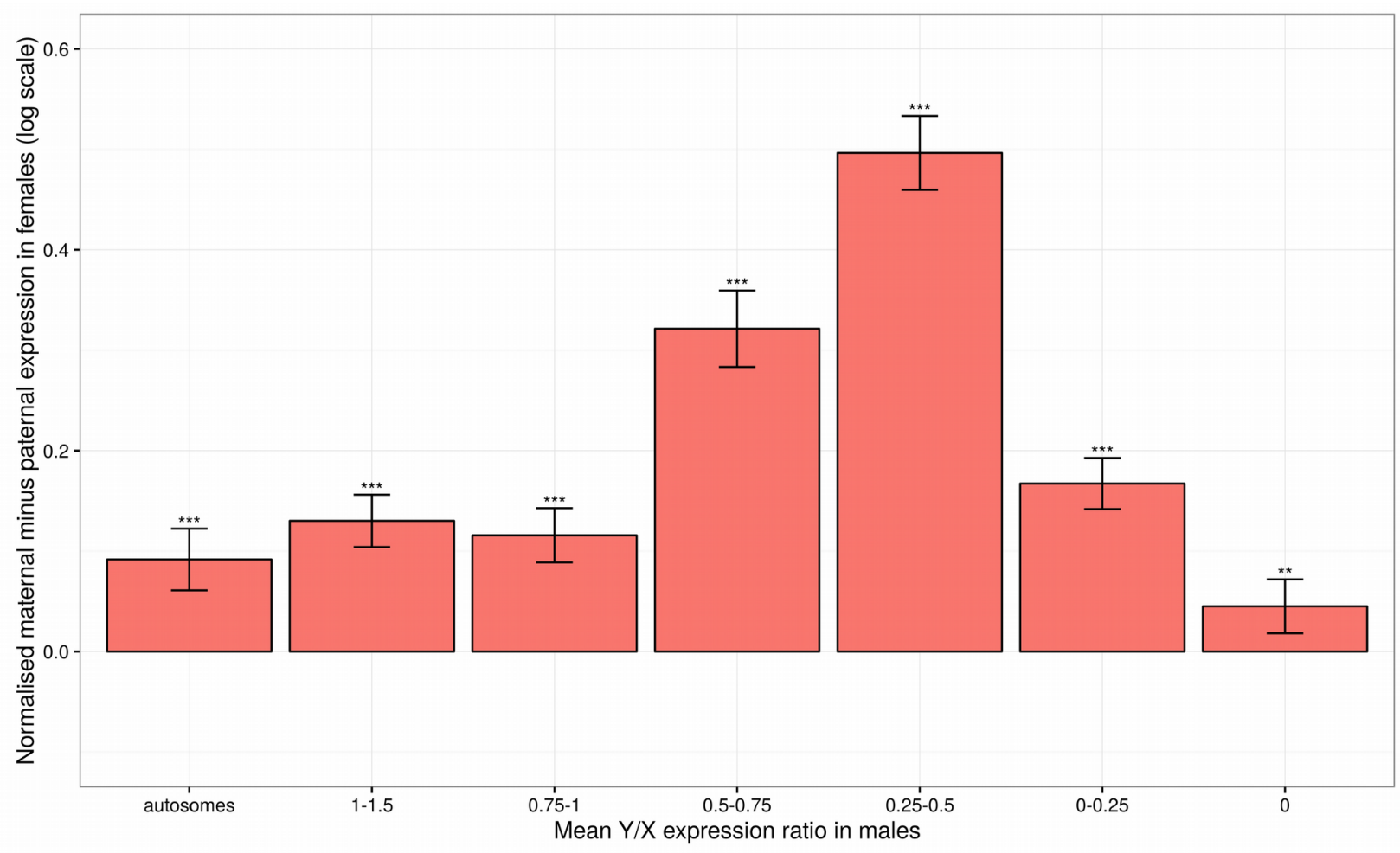

564 Supplementary Figure S10: Normalised expression difference between the maternal and

565 paternal allele in S. latifolia females in autosomal and sex-linked contigs for the leaf tissue. See

566 supplementary Figure S8 for legend and Supplementary Figure S4 for sample sizes for the 567 different contig categories. 


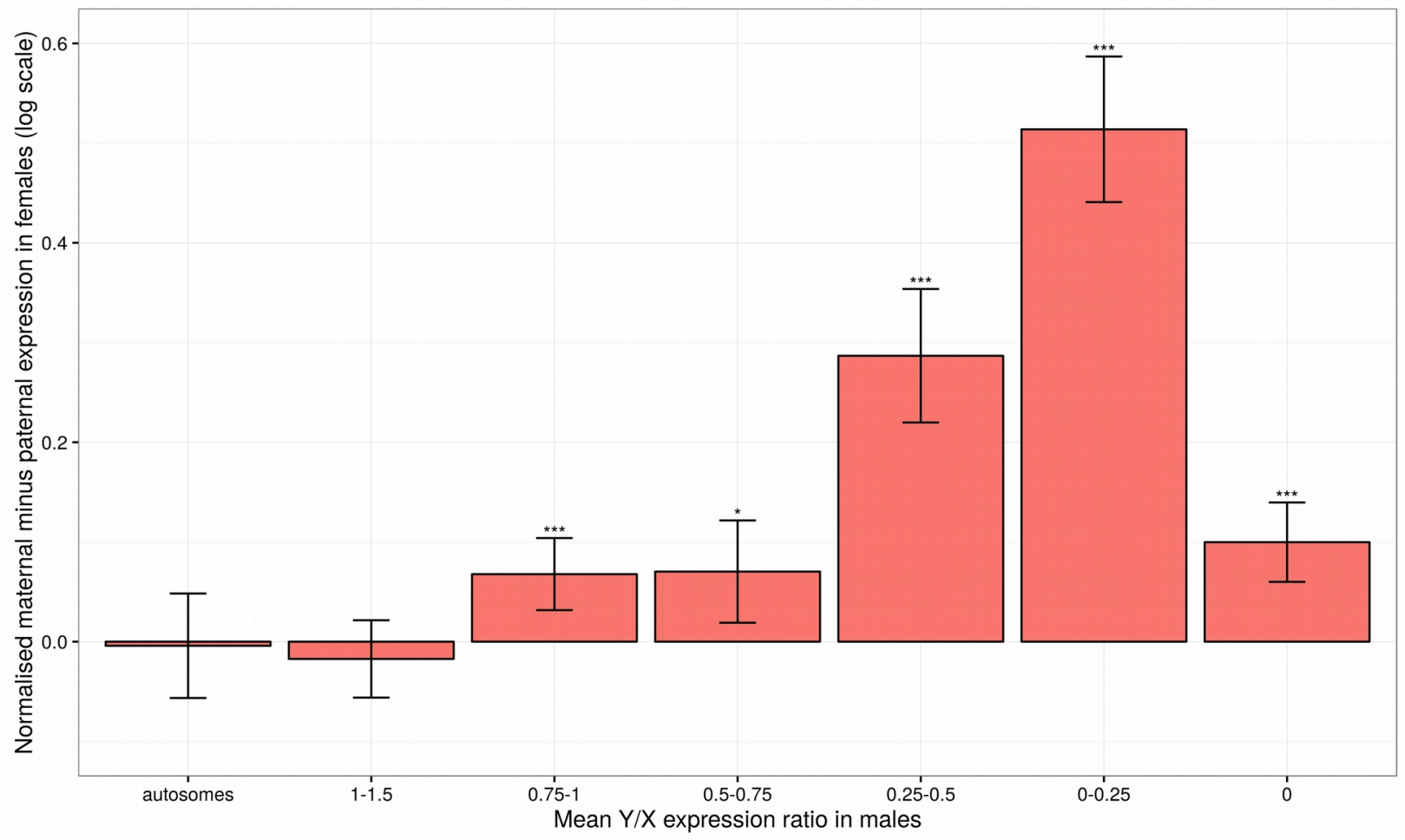

568 Supplementary Figure S11: Normalised expression difference between the maternal and 569 paternal allele in S. latifolia females in autosomal and sex-linked validated contigs for the

570 seedling tissue. See supplementary Figure S8 for legend and Supplementary Figure S5 for

571 sample sizes for the different contig categories. 


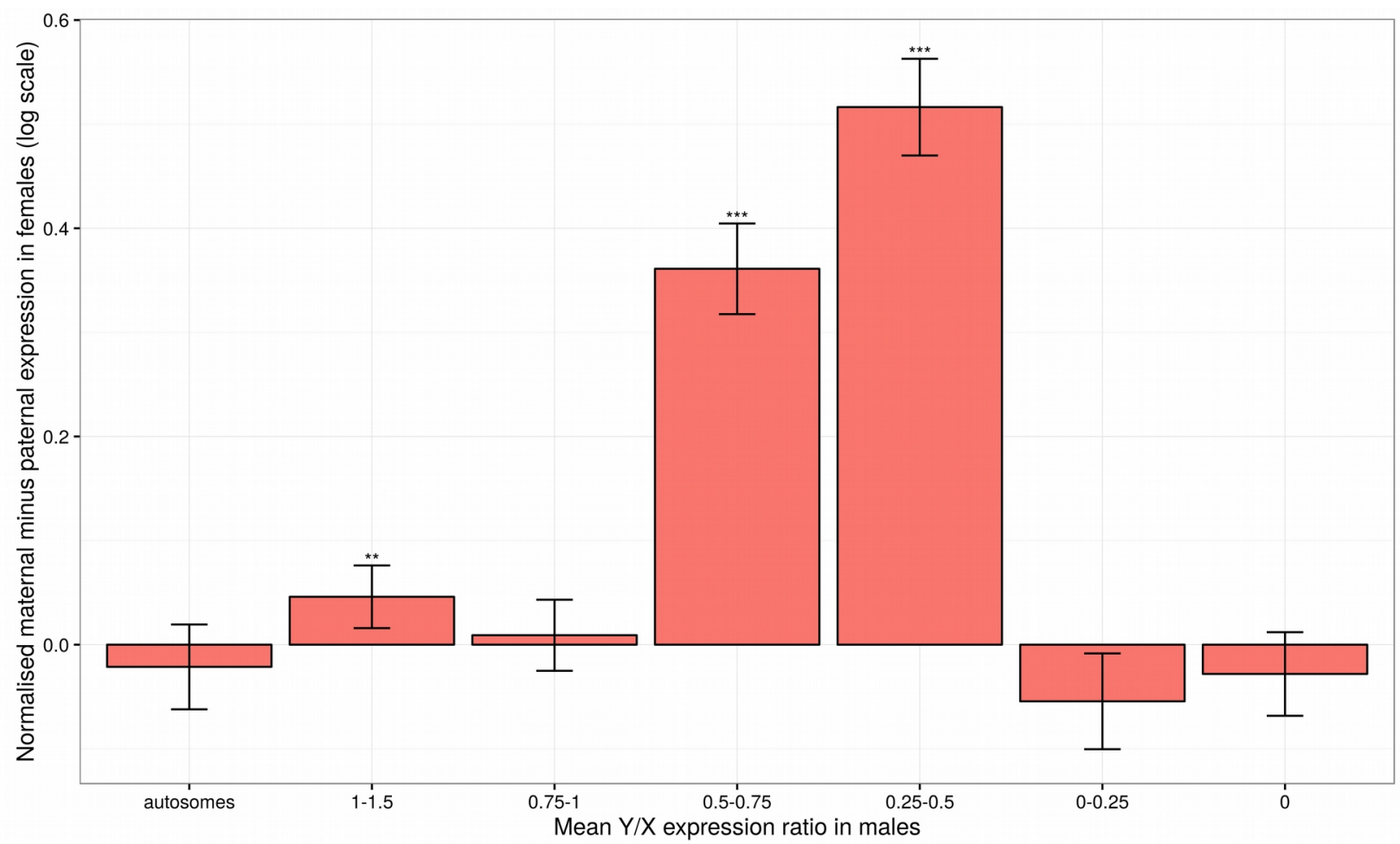

572 Supplementary Figure S12: Normalised expression difference between the maternal and 573 paternal allele in S. latifolia females in autosomal and sex-linked validated contigs for the

574 flower bud tissue. See supplementary Figure S8 for legend and Supplementary Figure S6 for 575 sample sizes for the different contig categories. 


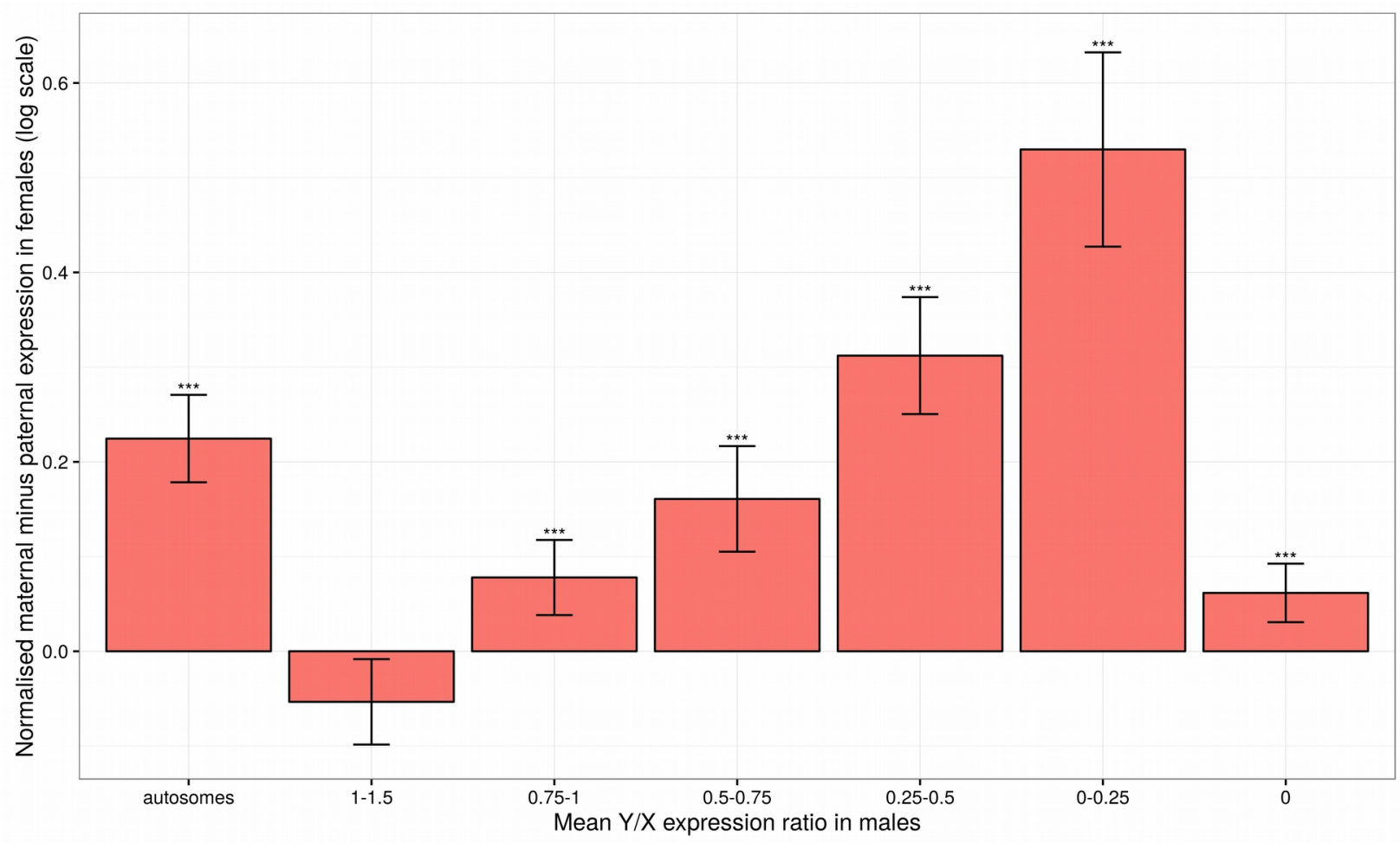

576 Supplementary Figure S13: Normalised expression difference between the maternal and

577 paternal allele in S. latifolia females in autosomal and sex-linked validated contigs for the leaf

578 tissue. See supplementary Figure S8 for legend and Supplementary Figure S7 for sample sizes

579 for the different contig categories. 

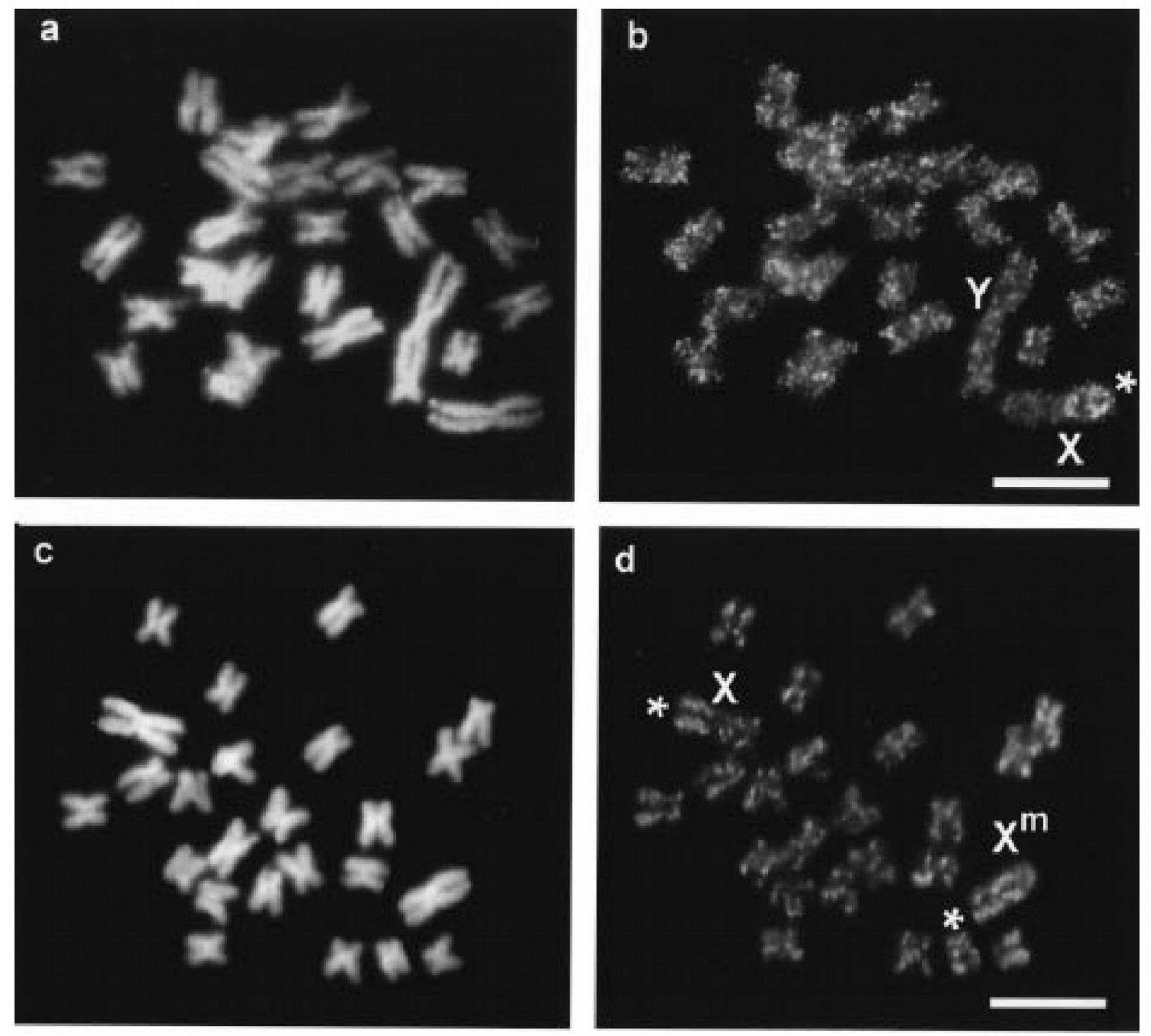

580 Supplementary Figure S14: Original DNA methylation staining results from Siroky et al

$5811998^{34}$. (a) Male metaphase chromosomes stained with PI. (b) FITC-anti-5-mC signals on the

582 same chromosomes. The hypomethylated shorter $\mathrm{X}$ arm is marked by an asterisk; The $\mathrm{X}$ and $\mathrm{Y}$

583 chromosomes are indicated. (c) Female metaphase chromosomes stained with PI. (d) FITC-anti-

584 5-mC signals on the same chromosomes. Shorter arms of the Xs are indicated by asterisks. The

585 hypermethylated $\mathrm{X}$ chromosome is marked as $\mathrm{X}^{\mathrm{m}}$. Bars $=5 \mu \mathrm{m}$. 


\section{Supplementary Tables}

587 Supplementary Table S1: library sizes (number of reads) of each individual and mapping

588 statistics.

589 Supplementary Table S2: Number of contigs after SEX-DETector inferences, removal of sex-

590 bias and selection of validated contigs in the three tissues.

\begin{tabular}{|l|c|c|c|}
\hline & \multicolumn{3}{|c|}{ Tissue type } \\
\hline & flower buds & leaves & seedlings \\
\hline number of ORFs & \multicolumn{3}{|c|}{46178} \\
\hline Unassigned & 33172 & 33564 & 33781 \\
\hline Autosomal & 11662 & 11558 & 11292 \\
\hline X/Y & 1140 & 772 & 844 \\
\hline X-hemizygous & 204 & 284 & 261 \\
\hline X/Y non sex-biased & 901 & 733 & 732 \\
\hline X-hemizygous non sex-biased & 103 & 275 & 205 \\
\hline X/Y non sex-biased validated & 339 & 345 & 365 \\
\hline X-hemizygous non sex-biased validated & 55 & 119 & 89 \\
\hline Autosomal validated & 74 & 79 & 77 \\
\hline
\end{tabular}

591 Supplementary Table S3: list of known sex-linked genes in S. latifolia and associated literature 592 references. 